\title{
Genome assembly and population genomic analysis provide insights into the evolution of modern sweet corn
}

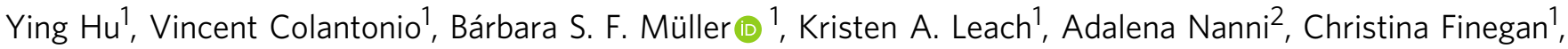
Bo Wang ${ }^{3}$, Matheus Baseggio (10 4,9, Carter J. Newton ${ }^{5}$, Emily M. Juht ${ }^{5,10}$, Lillian Hislop ${ }^{6}$, Juan M. Gonzalez (1) ${ }^{1}$, Esteban F. Rios (10 7, L. Curtis Hannah', Kelly Swarts ${ }^{8}$, Michael A. Gore (D) 4, Tracie A. Hennen-Bierwagen ${ }^{5}$, Alan M. Myers (i) ${ }^{5}$, A. Mark Settles (1) ${ }^{1,11}$, William F. Tracy ${ }^{6} \&$ Marcio F. R. Resende Jr. (iD ${ }^{1 \times}$

Sweet corn is one of the most important vegetables in the United States and Canada. Here, we present a de novo assembly of a sweet corn inbred line la453 with the mutated shrunken2reference allele (la453-sh2). This mutation accumulates more sugar and is present in most commercial hybrids developed for the processing and fresh markets. The ten pseudochromosomes cover $92 \%$ of the total assembly and $99 \%$ of the estimated genome size, with a scaffold N50 of $222.2 \mathrm{Mb}$. This reference genome completely assembles the large structural variation that created the mutant sh2- $R$ allele. Furthermore, comparative genomics analysis with six field corn genomes highlights differences in single-nucleotide polymorphisms, structural variations, and transposon composition. Phylogenetic analysis of 5,381 diverse maize and teosinte accessions reveals genetic relationships between sweet corn and other types of maize. Our results show evidence for a common origin in northern Mexico for modern sweet corn in the U.S. Finally, population genomic analysis identifies regions of the genome under selection and candidate genes associated with sweet corn traits, such as early flowering, endosperm composition, plant and tassel architecture, and kernel row number. Our study provides a high-quality reference-genome sequence to facilitate comparative genomics, functional studies, and genomic-assisted breeding for sweet corn.

\footnotetext{
${ }^{1}$ Horticultural Sciences Department, University of Florida, Gainesville, FL, USA. ${ }^{2}$ Department of Molecular Genetics and Microbiology, University of Florida, Gainesville, FL, USA. ${ }^{3}$ Cold Spring Harbor Laboratory, One Bungtown Road, Cold Spring Harbor, New York, NY, USA. ${ }^{4}$ Plant Breeding and Genetics Section, School of Integrative Plant Science, Cornell University, Ithaca, NY, USA. ${ }^{5}$ Roy J. Carver Department of Biochemistry, Biophysics, and Molecular Biology, lowa State University, Ames, IA, USA. ${ }^{6}$ Department of Agronomy, College of Agricultural and Life Sciences, University of Wisconsin-Madison, Madison, WI, USA ${ }^{7}$ Agronomy Department, University of Florida, Gainesville, FL, USA. ${ }^{8}$ Gregor Mendel Institute, Austrian Academy of Sciences, Vienna BioCenter, Vienna, Austria. ${ }^{9}$ Present address: Seneca Foods Corporation, LeSueur, MN, USA. ${ }^{10}$ Present address: Applied Plant Sciences Graduate Program, University of Minnesota, St. Paul, MN, USA. ${ }^{11}$ Present address: Bioengineering Branch, NASA Ames Research Center, MS 239-15, Moffett Field, CA, USA.

凶email: mresende@ufl.edu
} 
S weet corn (Zea mays L.) is grown all over the world and is one of the most important vegetables in the United States and Canada ${ }^{1}$. In the United States, sweet corn is a quintessential summer food, celebrated in local communities with festivals during harvest times. As a specialty crop, sweet corn has a farm gate value of $\$ 1.4$ billion per year, divided into fresh ( $\sim 4 \%$ of total value) and processing (canned and frozen corn) markets ${ }^{2}$. This starchy vegetable is a good source of dietary fiber, folate, niacin, essential amino acids, and lutein and zeaxanthin-two non-provitamin A carotenoids important in maintaining eye health and reducing the risk of age-related macular degeneration ${ }^{3}$.

Sweet corn is the result of mutations in genes involved in the starch biosynthesis pathway, which modify the carbohydrate composition by increasing sugar content in the endosperm while reducing starch content. Several genes in the starch biosynthetic pathway have been shown to increase sugar content in the endosperm when mutated ${ }^{4-8}$. Early commercial sweet corn hybrids increased sugar content by exploiting mutations in the sugary1 (su1) gene, a starch debranching enzyme. Due to this defective enzyme, the endosperm of a mature kernel will appear wrinkled and translucent (Fig. 1a). In the 1970s and 1980s, sweet corn breeders initiated the development of hybrids with mutations in the shrunken2 (Sh2) gene, which revolutionized the industry by improving eating quality and shelf life? .
The shrunken 2 gene encodes the large subunit of ADP-glucose pyrophosphorylase (AGPase), the first committed enzyme of the starch biosynthesis pathway ${ }^{10}$. Homozygous sh2 mutants accumulate more sugar, and the mature kernels are angular and shriveled due to reduced amounts of starch (Fig. 1a). Compared to homozygous sul mutants in which some sugars are converted to water soluble polysaccharides, homozygous sh 2 mutants have a higher sugar content at harvest, and this content has a slower rate of decline postharvest. Therefore, today, approximately $75 \%$ of the processing industry and nearly $100 \%$ of the fresh market industry utilizes hybrids containing the sh 2 mutation ${ }^{11}$.

In addition to the endosperm composition, commercial sweet corn hybrids are significantly different in phenotypic appearance from non-sweet maize hybrids, also known as field corn-one of the most widely produced cereals in the world. As a specialty vegetable, sweet corn plants have been selected for table quality traits such as kernel tenderness and color, and several esthetic traits that influence consumer acceptance, such as husk appearance, and silk and cob color. Furthermore, the plants are typically shorter, tend to flower earlier, and have a larger male inflorescence (tassel) than field corn ${ }^{12}$. Divergent selection between sweet and field corn predicts that a reference sweet corn genome will be considerably different than the field corn genomes previously assembled. To test this hypothesis, we sequenced a sweet corn inbred line, Ia453 with the $\operatorname{sh} 2-R$ allele (Ia453-sh2). Ia453-sh2 is a)
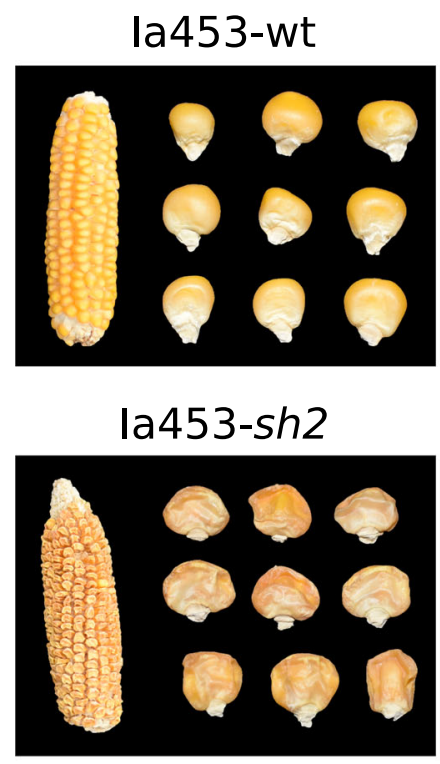

la453-su1

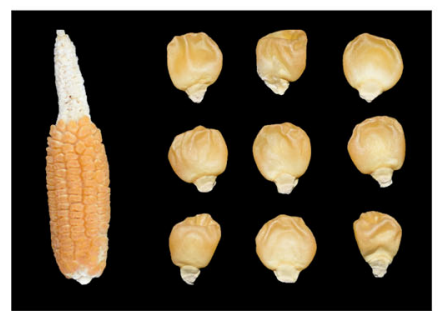

b)
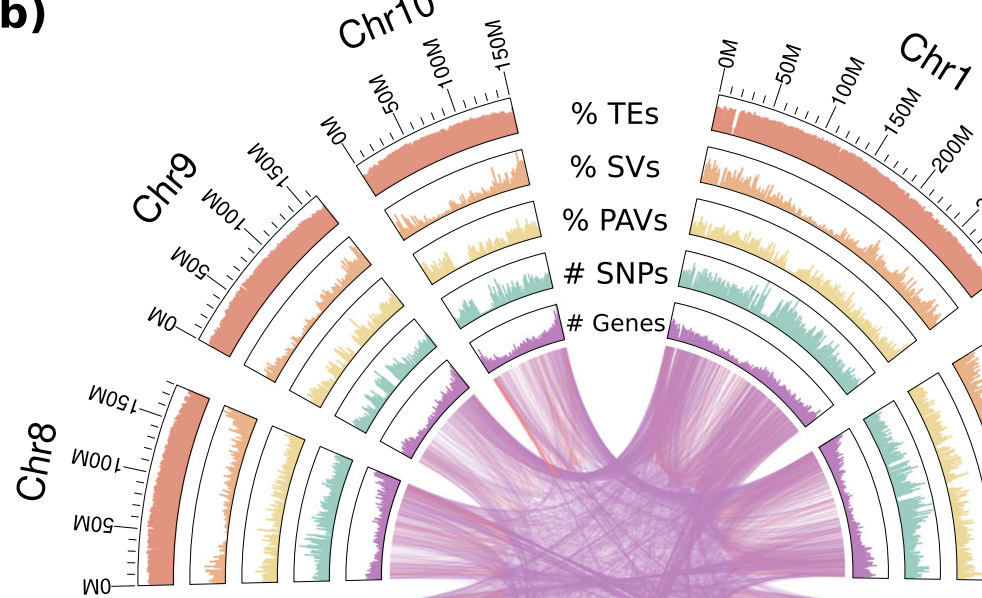

$i_{0}$

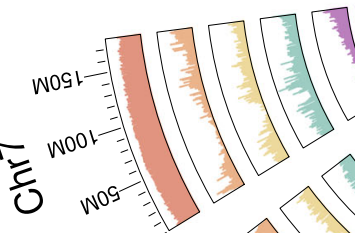

no

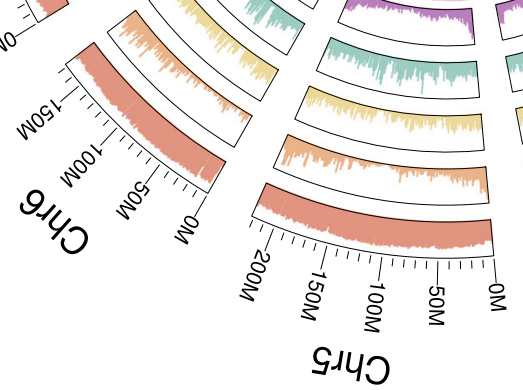

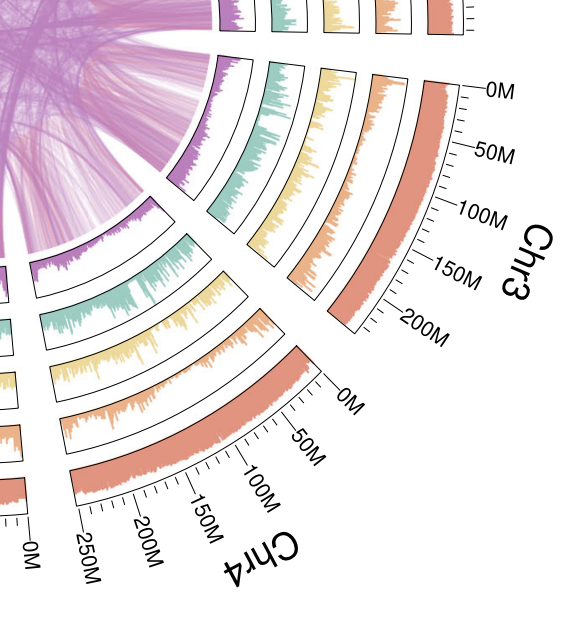

Fig. 1 Appearance of sweet corn in la453 isolines and la453-sh2 genomic features. a Visual ear appearance of sweet corn in la453 isolines, including wild-type (top), the su1 mutation (bottom), and sh2 mutation (center). b la453-sh2 genomic features. Outer-to-inner tracks indicate the following: transposable-elements (TEs) (fraction of TEs per $1 \mathrm{Mb}$ window); large structural variations (SVs) with length between 100 and 100,000 bps (fraction of SVs per $1 \mathrm{Mb}$ window) relative to B73 v4; presence and absence variants (PAV) (fraction of PAVs per $1 \mathrm{Mb}$ window) relative to B73 v4; single nucleotide

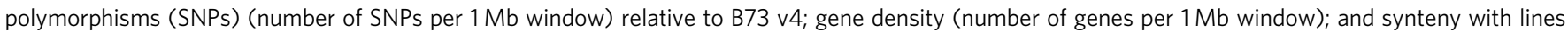
representing paralogous gene pairs between and within pseudochromosomes in la453-sh2 (purple and orange lines). 
an important public inbred line which contributed to the establishment of sh2 sweet corn and is one of the parents of Illini Chief and Illini Xtra Sweet, two of the first commercial sh2 sweet corn hybrids.

Here, we provide a high-quality reference genome of sweet corn through a combination of single-molecule real-time (SMRT) long-read sequencing, BioNano optical mapping, and Dovetail $\mathrm{Hi}-\mathrm{C}$ mapping technologies. Ia453-sh2 structural and genetic variations are identified through pairwise comparisons between Ia453-sh2 and six field corn genomes. Phylogenetic analysis of 5381 maize and teosinte genotypes reveals genetic relationships among teosinte, landraces, modern sweet corn, and a diverse set of field corn lines. Finally, population genomic analysis identifies regions of the genome under selection and candidate genes associated with sweet corn traits, such as early flowering, higher sugar content, and tassel architecture. Our study provides an important resource to facilitate comparative genomics, functional studies, and genomic-assisted breeding for sweet corn.

\section{Results}

Genome sequencing and assembly. Four data sources were used to assemble the Ia453-sh2 genome (Fig. 1b, Supplementary Fig. 1). First, $150.5 \mathrm{~Gb}$ ( 70-fold coverage, 19.9 million reads) of PacBio single-molecule long reads were self-corrected and assembled with Canu ${ }^{13}$, generating 15,550 contigs with an N50 of $0.39 \mathrm{Mb}$. BioNano optical maps were generated to anchor the PacBio contigs into 29 super scaffolds and 8486 unscaffolded contigs with an N50 of $120.9 \mathrm{Mb}$. To further anchor and orient the super scaffolds and unscaffolded contigs into pseudochromosomes, Dovetail Hi-C mapping was used for scaffolding through a hierarchical clustering strategy ${ }^{14}$. The Hi-C assembly was polished with ntEdit ${ }^{15}$ using short-read Illumina data. The final assembly has a genome length of $2.29 \mathrm{~Gb}$ and contains 10 long super scaffolds, hereafter denoted as pseudochromosomes, with a total length of $2.11 \mathrm{~Gb}$ as well as 8440 unassigned contigs with a total length of $177.23 \mathrm{Mb}$. The pseudochromosomes covered $92 \%$ of the total assembly and $99 \%$ of the estimated genome size based on $k$-mer analysis $(2.13 \mathrm{~Gb})$, with a scaffold N50 of 222.2 Mb (Table 1, Supplementary Table 1). Flow cytometry analysis estimated that Ia453-sh2 is $4.8 \%$ larger than B73 (Supplementary Table 2).

The quality and completeness of Ia453-sh2 genome was evaluated through BUSCO and long-terminal repeat (LTR) assembly index (LAI) analysis ${ }^{16,17}$. BUSCO analysis showed that $94.6 \%$ (1,363), $1.11 \%$ (18), and $4.09 \%$ (59) of the Plantae BUSCO genes are present in the assembled Ia453-sh2 genome as complete, fragmented, and missing genes, respectively. Out of the $94.6 \%$ complete genes, $88.05 \%$ were single-copy genes and $6.59 \%$ were duplicated genes. These results are similar to what was obtained for field corn reference genomes such as B73 v4 ${ }^{18}$,

\begin{tabular}{|c|c|}
\hline Genomic feature & la453-sh2 \\
\hline Length of la453-sh2 assembly (bp) & $2,285,829,126$ \\
\hline Length of 10 pseudochromosomes (bp) & $2,108,596,175(92.25 \%)$ \\
\hline Maximum scaffold length (bp) & $304,492,077$ \\
\hline Scaffold N50 (bp) & $222,201,399$ \\
\hline Number of unassigned contigs & 8440 \\
\hline Number of genes & 38,384 \\
\hline Number of genes in ten pseudochromosomes & $37,884(98.69 \%)$ \\
\hline Number of transcripts & 72,765 \\
\hline Genes with RNAseq support & 24,683 \\
\hline Total size of transposable elements (bp) & $1,689,995,319$ \\
\hline
\end{tabular}

W22 ${ }^{19}$, Mo17 ${ }^{20}$, F7, EP1, and DK105 genomes ${ }^{21}$ (hereafter denoted as field corn genomes) (Supplementary Table 3). The LAI score presents the proportion of intact LTR sequences in the genome and can be used to evaluate the assembly continuity and completeness (Supplementary Fig. 2). The Ia453-sh2 assembly resulted in a mean LAI score of 28.2 and was higher than the predominant field corn reference genome (B73 v4), indicating higher continuity and completeness of our assembly.

Protein-coding gene annotation. The protein-coding genes of the Ia453-sh2 genome were annotated by the MAKER-P pipeline $^{22}$ using RNA-seq and full-length cDNA data from seven different tissues of Ia453-sh2 as well as transcripts and proteins from eight published genome annotations as evidence (Supplementary Fig. 3). The combination of gene ab initio prediction tools AUGUSTUS ${ }^{23}$ and FGENESH ${ }^{24}$ were trained using AUGUSTUS databases "maize5" and "monocots" for gene prediction. After removing putative transposons and lowconfidence genes, 38,384 high-confidence protein-coding genes and 72,765 transcripts were identified. RNA-seq data supported $64 \%$ of the predicted genes. Nearly all $(98.69 \%)$ of the predicted gene models are located on the 10 pseudochromosomes, with only 500 gene models (1.31\%) located on unassigned contigs (Table 1).

Transposon annotation. Transposable elements (TEs) were annotated in Ia453-sh2 and the six field corn genomes (B73, Mo17, W22, EP1, F7, and DK105) with uniform methods to maintain consistency. Our pipeline identified a total of 2,647,709 TE elements in Ia453-sh 2 divided in 17 super-families. The TE elements were well distributed on the ten pseudochromosomes with a small increase in content near the centromeres (Fig. 1b, Supplementary Fig. 1, Supplementary Data 1). The TE annotation of Ia $453-$ sh 2 covered $1.69 \mathrm{~Gb}$, which represents $82.69 \%$ of the genome, including retrotransposon (69.85\%) and DNA transposon $(12.84 \%)$. The two most prevalent super-families were both long terminal repeat (LTR) retrotransposons, consisting of 843,793 (784 Mb total) Gypsy elements and 456,321 (420 Mb total) Copia elements. The 418,078 terminal inverted repeat (TIR) elements identified account for $140 \mathrm{Mb}$, while Helitrons were predicted to cover $104 \mathrm{Mb}$ of the genome in 350,444 elements. Compared to all six field corn genomes, Ia453-sh 2 contains the lowest number of TEs and the lowest percentage of the genome length covered by TEs (Supplementary Data 1). However, the fraction of Ia453-sh 2 covered by LTR-Copia-like retrotransposon (20.58\%), TIR Pif/Harbinger (0.56\%), and MITE Tc1/Mariner $(0.08 \%)$ represented the largest percentages compared to all six field corn genomes, with a relative increase in the percentage of each family ranging from 12 to $23 \%$ compared to the average content in the field corn genomes (Supplementary Data 1). These analyses illustrate the high level of divergence in TE families between maize lines, which results in structural and sequence variation of protein-coding genes and their regulatory sequences.

Structural variation of $\boldsymbol{s h} 2-\boldsymbol{R}$ allele. The $\operatorname{sh} 2-R$ allele is a loss-offunction allele that conditions high sugar content at eating stage and propelled the modern sweet corn industry. At least two structural rearrangements occurred in $\operatorname{sh} 2-R$ relative to the Sh2 allele in field corn. In Ia453-sh2, the first half of the gene was separated by a $5.45 \mathrm{~kb}$ Copia LTR retrotransposon, which is intact, conserved, and young (insertion time estimated as zero due to conserved sequence) (Fig. 2a, Supplementary Table 4). The second half of the gene is inverted and separated from the first half by $49.44 \mathrm{~kb}$ of TE sequence, including one intact Copia LTR retrotransposon, one intact Gypsy LTR retrotransposon, and a 
a)

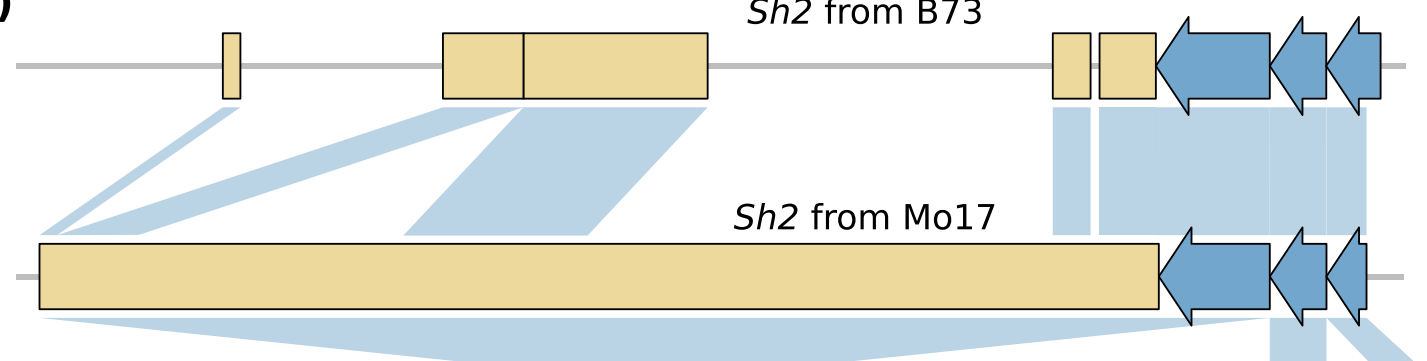

Sh2 from B73

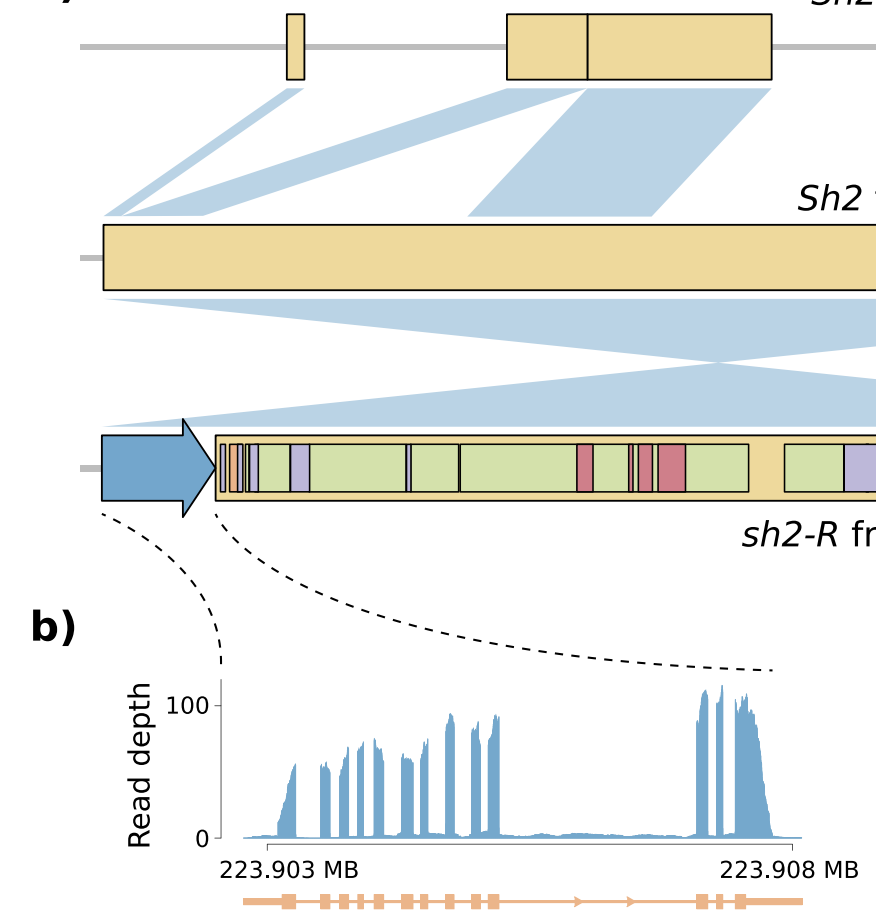

Zm00045a021195

\section{sh2-R from la453-sh2}

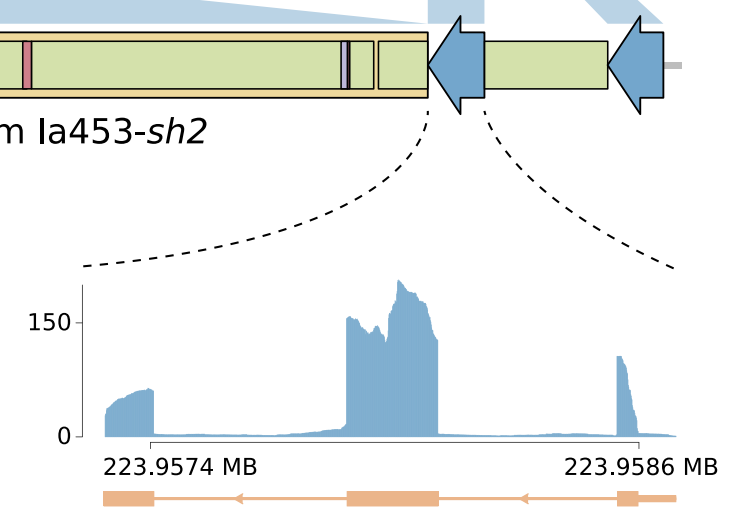

Zm00045a021196

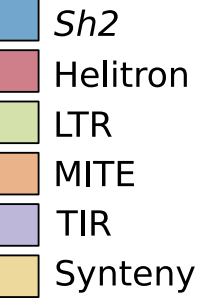

Synteny

c)

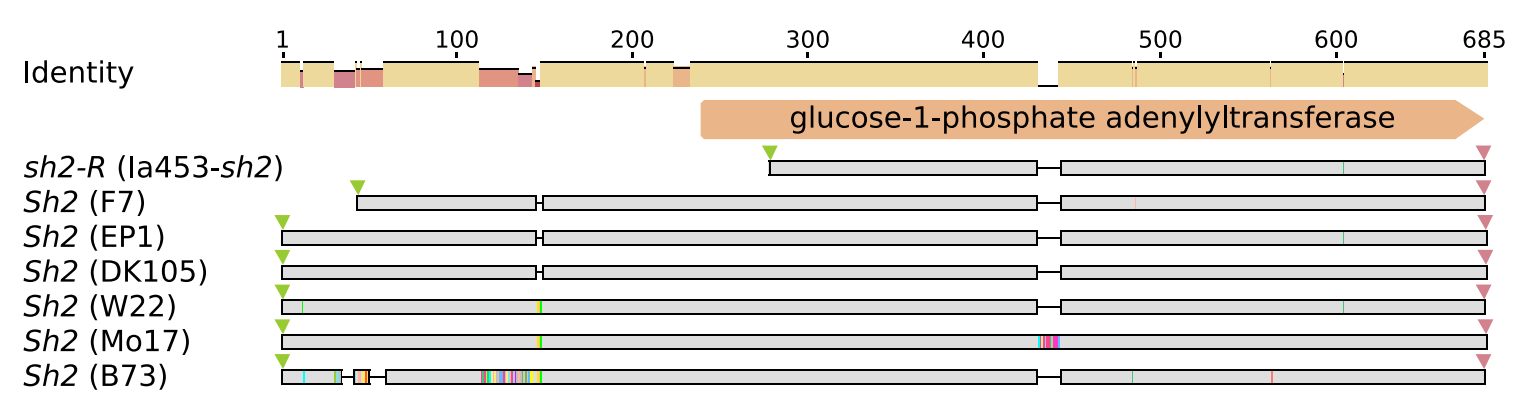

Fig. 2 Structural variation of the sh2-R allele. a Scheme of the structural variations between Sh2 in B73 and Sh2 in Mo17 (the top pairwise comparison) as well as Sh2 in Mo17 and sh2-R in la453-sh2 (the bottom pairwise comparison). The Sh2 in B73 and Mo17 is drawn the same size as the sh2-R allele in la453sh2 (blue arrows). Features within each allele are drawn to scale. The syntenic regions were drawn as yellow boxes and connected using blue wedges. The annonated TE elements are only shown in la453-sh2 for simplicity. Two insertion sequences in the sh2-R allele contain a variety of predicted TE elements. b Two predicted gene models annotated in the sh2-R loci and their expression profile represented as the number of reads per Gb of total RNA-seq aligned reads. c Comparison of the predicted amino acid sequences of sh2-R (Zm00045a021195) from la453-sh2 with Sh2 from six field corn lines, using the Geneious multiple sequence alignment. Conserved amino acids are marked in gray, gaps are indicated as lines and differences are highlighted with different colors. The identity panel displays the identity across all amino acid sequences for every position. Small green and red triangles are indicating start and stop codons, respectively.

variety of predicted TE events. This large TE sequence is present downstream of $\operatorname{Sh} 2$ in Mo17 with $99.6 \%$ similarity but partially absent in B73 (Fig. 2a). This results supports the intrachromosomal inversion proposed by Kramer et al. ${ }^{25}$, and here we show that this inversion happened in the middle of the gene (Fig. 2a). The insertion of the two intact LTRs in the $49.44 \mathrm{~kb} \mathrm{TE}$ sequences was estimated to occur about 0.29 and 0.58 million years ago, but these insertions likely preceded the inversion (Supplementary Table 4). The inversion causes two separate gene models to be predicted in the sh2- $R$ locus (Zm00045a021196 and Zm00045a021195) (Fig. 2b). Zm00045a021196 includes the fourth Sh2 exon as well as two additional exons with gene expression support. Zm00045a021195 includes the last 13 exons of Sh2. Both genes are expressed in the endosperm, but $\mathrm{Zm00045a021195}$ encodes an incomplete glucose-1-phosphate adenylyltransferase domain missing 36 amino acids in the $\mathrm{N}$ terminus of the conserved domain (Fig. 2c).

Global genomic comparison of Ia453-sh2 to field corn genomes. A pairwise genomic comparison was performed between Ia453-sh2 and six field corn genomes. One-to-one pairwise genome alignment resulted in an average syntenic match of $68.95 \%$. The three flint (F7, EP1, and DK105) genomes had a higher percentage of syntenic matches $(69.86 \%)$ compared to dent (B73, Mo17, and W22) genomes (68.04\%) (Supplementary Table 5). In addition, in those one-to-one aligned regions, we identified an average of 10,493,202 SNPs between Ia453-sh2 and the surveyed field corn genomes (Supplementary Table 5). The number of small insertions and deletions (indels $<100 \mathrm{bp}$ ) in those one-toone aligned regions averaged 806,274 and accounted for about 
Table 2 Summary of the la453-sh2- and B73-specific genes compared to other six corn genomes.

\begin{tabular}{|c|c|c|c|c|c|c|c|c|}
\hline Genes specific to & Number of genes calculated from the pairwise comparison & la453-sh2 & B73 & Mo17 & W22 & DK105 & EP1 & F7 \\
\hline la453-sh2 & Genes in la453-sh2-specific regions & - & 364 & 425 & 455 & 387 & 357 & 403 \\
\hline \multirow[t]{3}{*}{ B73 } & Genes in B73-specific regions & 340 & - & 423 & 455 & 383 & 369 & 420 \\
\hline & Genes from B73 with low identity or absent for the pairwise comparison & 827 & - & 941 & 1102 & 926 & 911 & 975 \\
\hline & Overlap & 122 & - & 161 & 186 & 115 & 115 & 143 \\
\hline
\end{tabular}

$0.2 \%$ of the Ia $453-$ sh 2 genome (Supplementary Table 5). Notably, there were fewer SNPs and small indels between DK105 and Ia453-sh 2 than other comparisons, suggesting a closer relationship between these two lines. The Ia453-sh2 specific regions were also detected by comparing Ia453-sh 2 and other six field corn genomes using a sliding-window method. We identified between $56-61 \mathrm{Mb}$ sequences that are specific in Ia453-sh2 compared to B73, Mo17, W22, EP1, F7, and DK105, accounting for $2.5 \%$ of the genome. An overlap of these unique regions with our TE annotation indicated that $68-69 \%$ of the specific sequences were found to overlap with TEs in all six comparisons (Supplementary Table 5). Large structural variations (SV) (100-100,000 bp) were detected with an average number of 23,664 compared against the field corn genomes, which encompassed, on average, a total length of $351.59 \mathrm{Mb}$ (Supplementary Data 2). Repeat contraction or expansions made up the majority of SV sequences (88-90\%) in all six comparisons (Supplementary Data 2).

Pan-gene and gene structural variation analysis. The distribution of orthologous gene families in Ia453-sh2 and all six field corn genomes was defined using OrthoMCL ${ }^{26}$. We identified an average of 22,322 core genes belonging to gene families that were shared by all seven corn genomes, 16,667 dispensable genes missing orthologous counterparts in at least one of the tested genomes, and 5545 singleton genes assigned to families which were unique to only one line (Supplementary Fig. 4). Out of the set of 22,322 core genes, we identified a set 7864 highly conserved protein coding genes using pairwise comparative analysis of the sweet corn genome with each of the six field corn genomes. These genes were functionally enriched in conserved biological processes and molecular functions, such as pre-mRNA $5^{\prime}$-splice site binding (GO:0003843, $p$ value $=1.1 \mathrm{e}-4$ ) or photoreactive repair (GO:0000719, $p$ value $=8.4 \mathrm{e}-4$ ) (Supplementary Data 3$)$. This pairwise comparative analysis also resulted in a set of Ia453-sh2 specific genes that could not be aligned to other field corn genomes or that were aligned with very low coverage and identity (Supplementary Fig. 5). We identified, on average, 148 genes specific to Ia453-sh 2 when individually compared with each field corn genome (Table 2), with six of these genes found uniquely in the Ia453-sh2 genome (Supplementary Table 6). The same analysis in B73 resulted in similar number of genes unique to B73 (Table 2).

Among the six Ia453-sh2-specific genes, Zm00045a011525 encodes an acetylating enzyme methylmalonate-semialdehyde dehydrogenase, a gene previously found to affect seed storage reserve and germination rate $^{27}$. Zm00045a021614 encodes an Fbox, LRR, and FBD domain containing protein, which plays a role in plant immune responses through involving in hormone pathways or functioning in plant-pathogen interactions ${ }^{28,29}$. Zm00045a046064 encodes a PAE1 proteasome subunit alpha type-5, which is involved in protein and RNA degradation, and is associated with the plant response to the majority of stresses ${ }^{30}$.

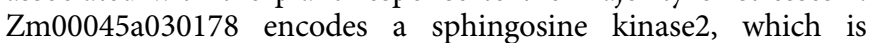

involved in the production of sphingolipid metabolites and abscisic acid (ABA) signaling that mediates stomatal closure, inhibition of seed germination, and root elongation ${ }^{31}$. The other two genes ( $\mathrm{Zm00045a028561}$ and $\mathrm{Zm00045a030528)}$ are predicted to encode proteins with unknown functions. None of those six genes had premature stop codons and all of them had proper annotations that agree with aligned ESTs from other species, and protein homology data based on annotation edit distance (AED) score (AED: 0.11-0.42). Furthermore, the MAKER mRNA quality index (QI), another quality metric to dissect the gene annotation,

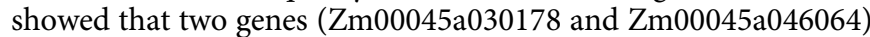
had support from splice-sites confirmation (QI2 $=0.85$ and 0.50 , respectively). In addition, these two genes were expressed in kernel, leaf, stem, silk, husk, ear, and pollen tissues, while the other four genes were not detected to be expressed in the same tissues (Supplementary Fig. 6). These results were further supported by the analysis of publicly available $3^{\prime}$-RNA-seq ${ }^{32}$ from five sweet corn (P39, Il14H, Il677a, Il101T, and IA2132) and five field corn inbred lines (B73, Mo17, W22, EP1, and F7) where both genes were only expressed in sweet corn (Supplementary Fig. 6). Seed germination and response to biotic stress are traditionally important traits in sweet corn breeding, which could explain the presence of these two genes with detected gene expression. Further studies are required to validate their function and test if they are present in a broad sweet corn germplasm.

Phylogenetic analysis traces the evolutionary origins of sweet corn. We evaluated genotypes of diverse maize accessions using a genotyping-by-sequencing build to help understand how sweet corn is related to other types of maize. The panel included teosinte, landraces, and field corn inbred lines in addition to 822 sweet corn lines. Leveraging RaXML-NG, we produced a maximum likelihood-based phylogenetic tree comprising 5381 diverse maize accessions (Fig. 3a). The tree was rooted with the teosinte Zea luxurians [Durieu and Asch.]. As expected, we found other teosintes including Z. mays ssp. mexicana [Schrad.], $Z$. mays ssp. parviglumis [Iltis and Doebley], and Z. luxurians to be sister clades to cultivated maize. Maize accessions from Central Mexico, Northern Mexico, US Southwest, and US Puebloan were found to subsequently diverge. The sweet corn accessions were primarily found in their own clade sister to the remaining maize populations. This is likely due to breeding of sweet corn specific traits, and the large contribution of a few sweet corn genotypes, such as Golden Bantam and Stowell's Evergreen, to modern commercial varieties. Flint corn varieties including F7 and DK105 were found to be grouped with the sweet corn clade, supporting the hypothesis that modern sweet corn varieties share the origins or are primarily derived from Northern Flints ${ }^{33}$, a race of corn grown in the past in eastern North America. Clustering of the samples using STRUCTURE and discriminant analysis of principal components (DAPC) also showed the separation of sweet corn from the rest and further divided the sweet corn clade into different groups (Fig. 3c, d, 
a)

d)

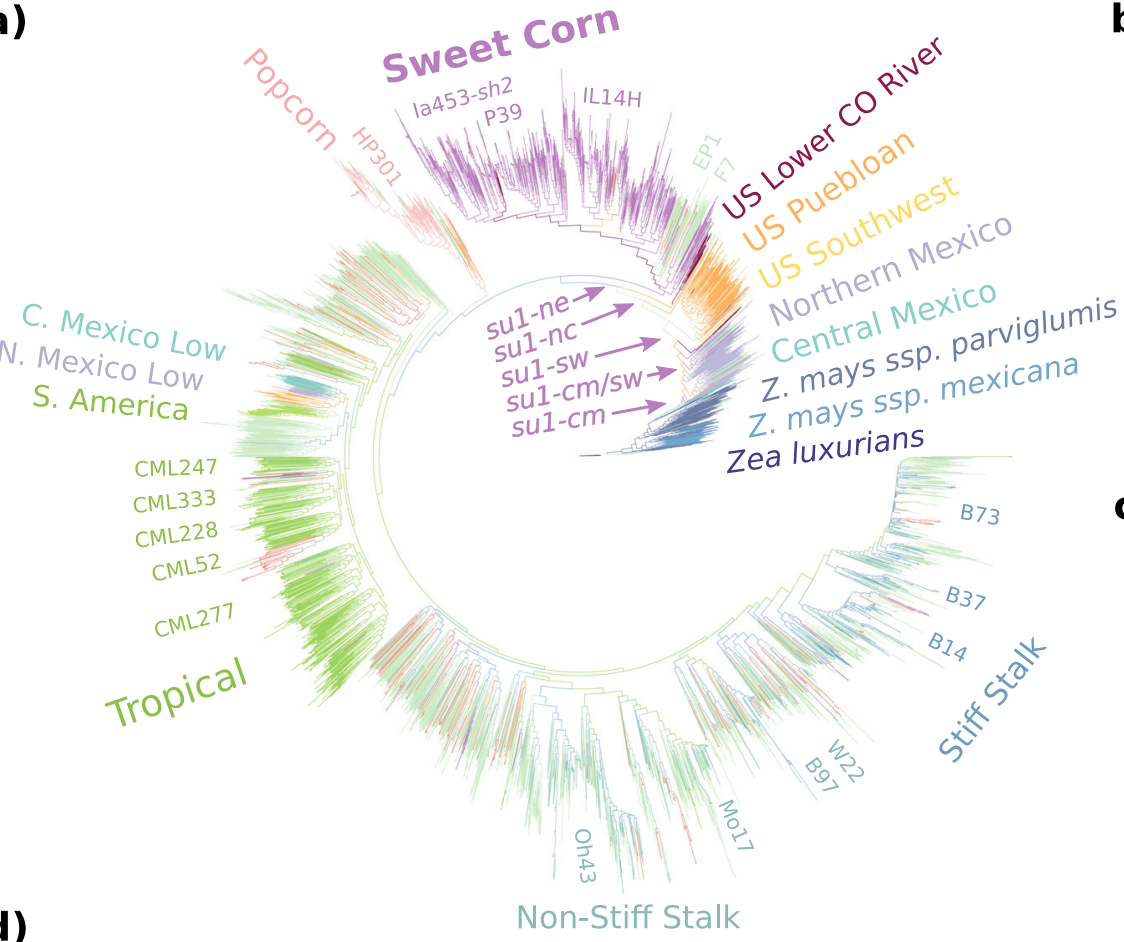

b)

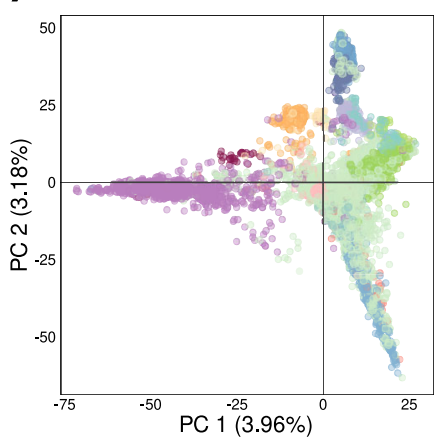

c)

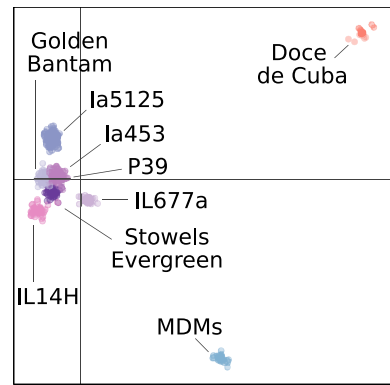

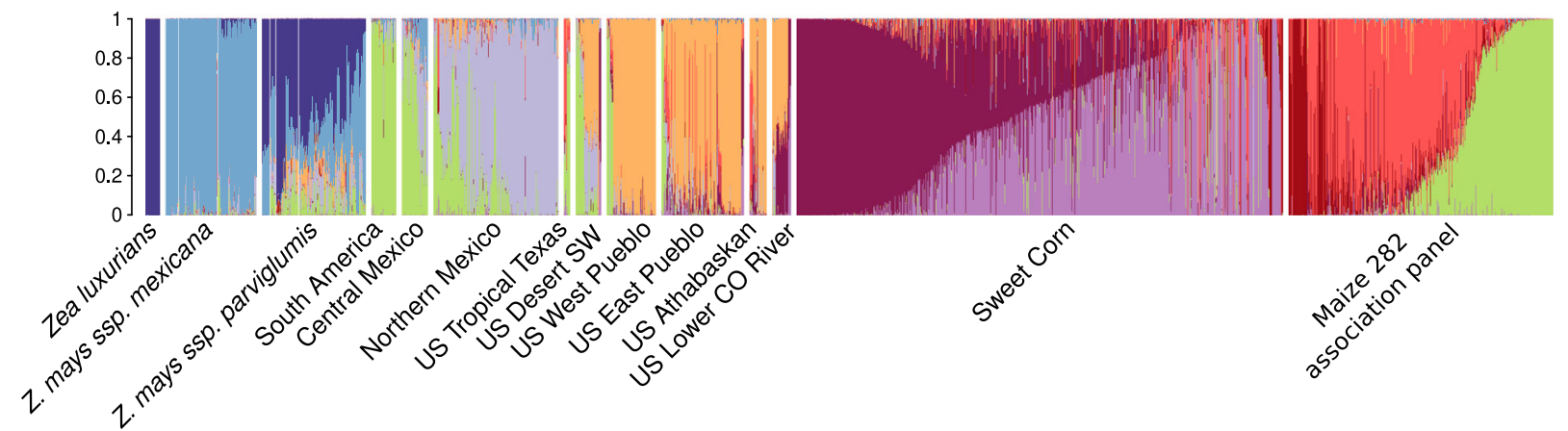

Fig. 3 Phylogenetic relationships and population structures of diverse teosinte and maize accessions. a Phylogenetic tree of teosinte, landraces, sweet corn, and field corn genotypes. b Principal component analysis color-coded as in the phylogenetic tree. c Discriminant analysis of principal components (DAPC) plot with only the sweet corn lines using different colors, highlighting an important line that is representative of each cluster-"MDMs" label represent a group of sweet corn lines bred for Maize Dwarf Mosaic Virus. d Bayesian clustering of the population using STRUCTURE with $K=9$. Sweet corn and Maize 282 association panel were sorted based on one of their prevalent populations. The other groups were sorted based on the altitude where they were grown according to Swarts et al. ${ }^{57}$. The colors used do not represent the same grouping as panels (a) and (b).

Supplementary Fig. 7). STRUCTURE analysis with different group numbers ( $k=4$ to 16$)$ was performed (Fig. 3d, Supplementary Fig. 7). The optimum number based on Evanno's criteria was estimated as nine with well-defined groups for $Z$. luxurians, Z. mexicana, and landraces from South America, Northern Mexico, and US Puebloan. The marker set used was not able to allocate Zea parviglumis into its own group, presumably due to missing data or undersampling of genetic variability. The STRUCTURE cluster that contained the majority of sweet corn lines is represented with high membership probability $(>0.99)$ by the variety Golden Bantam and inbred line P39, two important genotypes in the history of sweet corn breeding. A second large STRUCTURE cluster traces back with high membership probability to IL677a, a sul line which is also the source of the mutant gene sugary enhancer $(s e)^{34}$, a third mutant gene used in sweet corn breeding programs and recently cloned ${ }^{35}$. A DAPC calculated using only the sweet corn genotypes supported a cluster containing IL677a and further identified groups that contained additional lines known to have contributed to modern sweet corn breeding, such as Stowell's Evergreen and Ia5125 $5^{4-8}$ (Fig. 3c).

From an evolutionary perspective, sweet corn is known to have arisen multiple times as evidenced by the presence of multiple independent sul alleles ${ }^{33}$. We identified in the phylogenetic tree the samples containing the alleles identified by Tracy et al. ${ }^{33}$ to infer further the evolutionary history of sul genotypes (Fig. 3a). We found the Maiz Dulce variety Guanajuato, containing the su1$\mathrm{cm}$ allele with a $1.3 \mathrm{kbp}$ TE inserted in exon 1 , to be grouped with the central Mexico landraces. The Ducillo de Noroeste variety from Sonora containing the su1-sw (N561S) allele was found to be clustered with the northern Mexico landraces. The southwestern 12 Row varieties, which included Tawa'ktci, Moencopi, and Hotevilla, were found to be clustered with Puebloan landraces from the southwestern United States. These landraces also contain the su1-sw allele suggesting a migration from northern Mexico to southwestern United States. The Nueta varieties derived from the Great Plains Flints and Flours containing the su1-nc (R504C) allele were found to be diverging near the flint 
a)

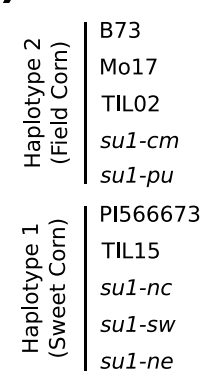

c)
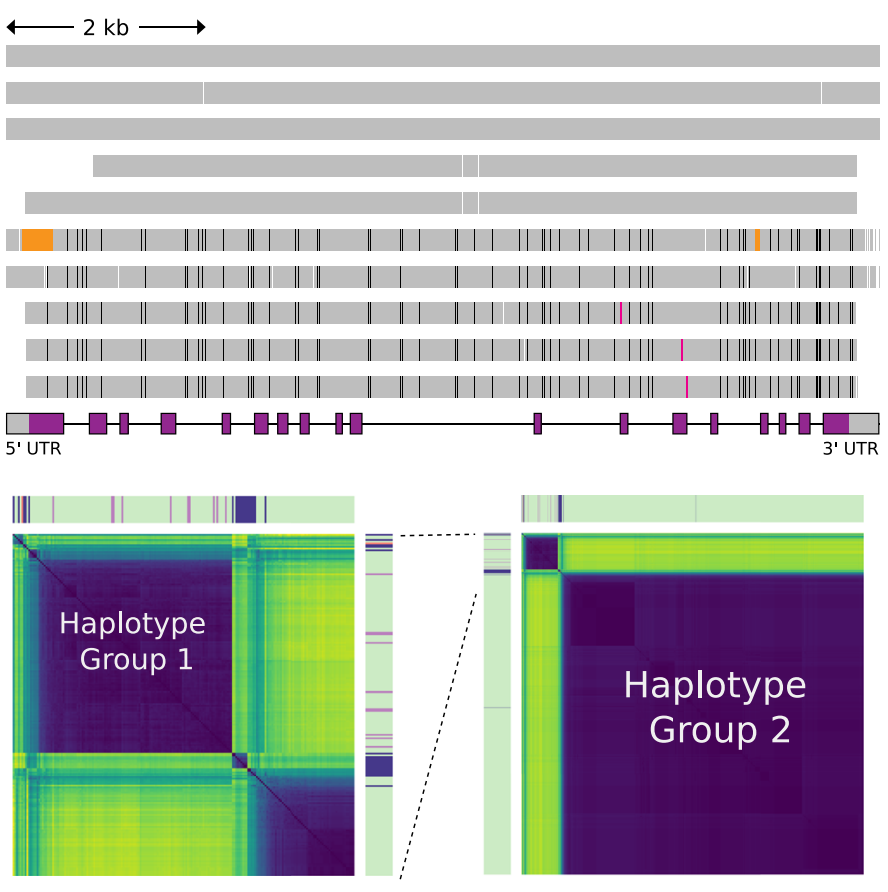

b)

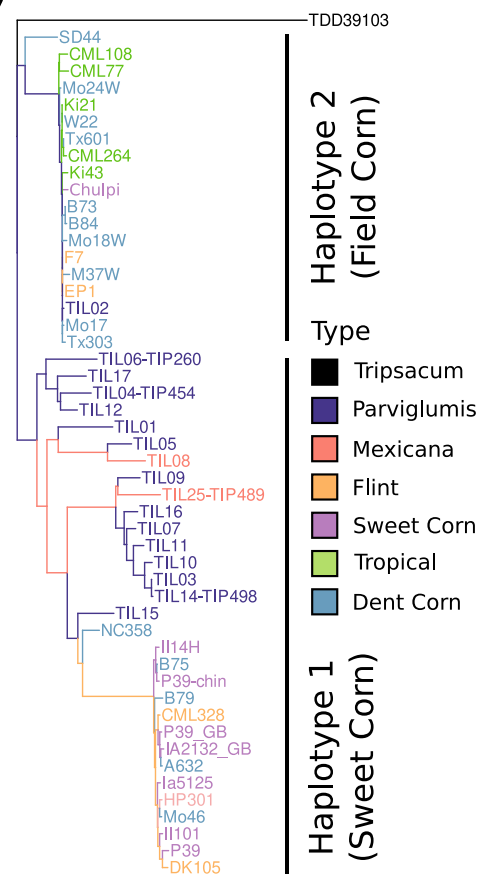

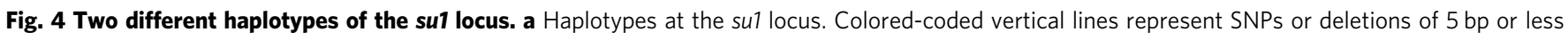
relative to the B73 reference sequence. Black lines denote SNPs present in su1-ne, white lines indicate SNPs in other alleles not shared with su1-ne, magenta lines indicate causative agents of the su1-defect, and orange blocks indicate deletions in PI566673 ${ }^{115}$ (Zea mays ssp. mexicana) relative to B73. Every

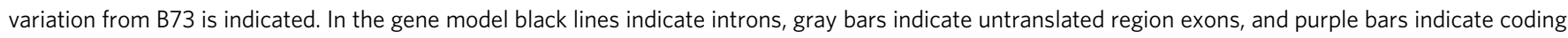
sequence exons. The figure is drawn to scale except for a few SNP locations that have been slightly adjusted to allow visual resolution. The sequences of regions not covered by gray bars were not determined. b Neighbor-joining su1 tree rooted in Tripsacum dactyloides, containing teosinte samples and

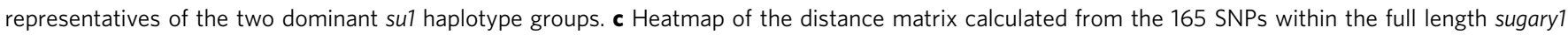
gene. The left part of the panel represents a blowup of the full heatmap, presented on the right corner of the same panel. Haplotype group 1 is referred in the text as the sweet corn haplotype while haplotype 2 is the field corn haplotype.

varieties at the base of the sweet corn clade. Finally, the sugary1reference 36 allele (also known as su1-ne-W578R) was placed in the group that contained the majority of sweet corn lines, as expected given that most modern sweet corn lines contain the su1-ne allele 33 .

We further explored the sul locus and identified the presence of two distinct groups of sequences for sul, hereafter denoted as major haplotype groups. While the five known alleles have independent origin ${ }^{33}$, as evidenced by distinct causative mutations, three of such su1 alleles (su1-nc, su1-sw, su1-ne) are contained in one of the major haplotype group (referred as the "sweet corn" haplotype) that includes 165 SNPs relative to B73 (Fig. 4). These three alleles were the ones involved in the origins of sweet corn in North America. The other two sul sweet corn alleles, $s u 1-p u$ in the Andean landrace "Chullpi" 37 and $s u 1-c m$ in the Maiz Dulce line from central Mexico, exhibit very few SNPs relative to B73 and thus contain the "field corn" haplotype of the su1 locus (Fig. 4a). Extending this analysis to publicly available genomic sequences revealed distinct phylogenetic separation of the two haplotype groups (Fig. 4b). A k-means clustering of the B73 sugary1 sequence updated with HapMap $3 \mathrm{SNPs}^{38}$ shows that 114 lines have the "sweet corn" haplotype and 1065 have the "field corn" haplotype (Fig. 4c). The former group includes, in addition to most sweet corns, genotypes from teosinte (both $Z$. parviglumis and $Z$. mexicana), popcorn, flint lines, and a minority of field corn lines, whereas most field corn lines are represented by the second haplotype group (Fig. $4 \mathrm{~b}, \mathrm{c}$ ). The $Z$. parviglumis genotype TIL02 was the only teosinte line assigned to the field corn haplotype group (Fig. 4a, b). Nucleotide diversity among corn samples was low in general, but particularly lower in Haplotype 2
$\left(\Pi_{\mathrm{HAP} 1}=0.00092 ; \Pi_{\mathrm{HAP} 2}=0.00024 ; 11,598 \mathrm{bp}\right)$, suggesting evidence of selection in this locus. Out of the landraces sequenced in HapMap3, the "sweet corn" haplotype is also present in Assiniboine (BKN014), Longfellow Flint (BKN016), Tabloncillo (BKN035), Poropo (BKN010), and Reventador (BKN022) (Supplementary Fig. 8). Interestingly, Reventador, a popcorn landrace from Mexico has been hypothesized to be one of the parents of the sweet corn landrace Ducillo del Noroeste and is also thought to be the ancestor of the Tabloncillo race $^{39}$. Altogether, these results suggest a common origin in northern Mexico for modern sweet corn in the United States. prior to the natural creation of the three different su1 alleles.

Population genomic analyses reveal sweet corn specific selective sweeps. In addition to having low starch content, sweet corn varieties are morphologically and physiologically distinct from field corn. They are shorter, have larger tassels, flower earlier, and have droopier leaves. Sweet corn plants also tend to have more tillers, and a major QTL, tin1, associated with tillering growth habit of the sweet corn was recently reported ${ }^{40}$. In addition, sweet corn has been selected upon by breeders for different traits, including processor and consumer-oriented traits such as shape, size, flavor, and kernel row number. Population genomic analyses were used to scan the genome for signatures of selection with the hypothesis that these may contribute to characteristic sweet corn phenotypes. We calculated fixation index $\left(F_{\mathrm{ST}}\right)$, Tajima's $D$ in 10 $\mathrm{kb}$ windows, and cross-population composite likelihood ratio (XP-CLR) as metrics of population differentiation between sweet corn populations (su1-type, sh2-type, and all sweet corn) and the maize 282 association panel. 


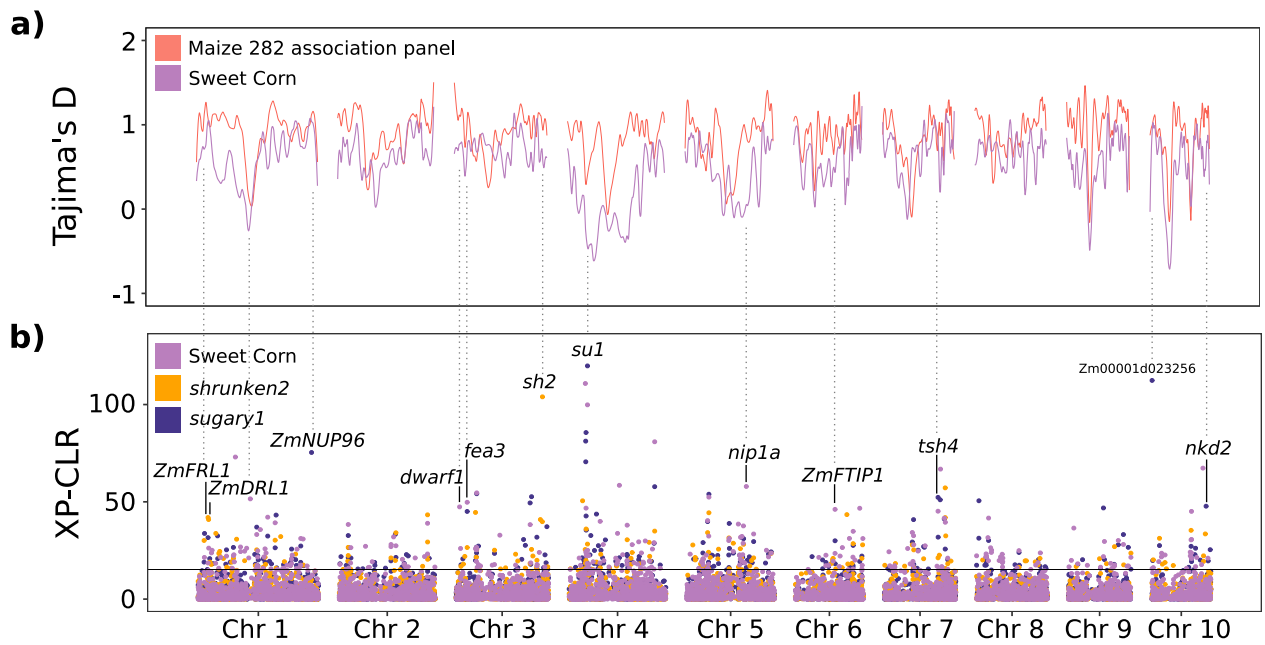

Fig. 5 Genome-wide distribution of sweet corn specific selective sweeps. a Tajima's $D$ calculated on the sweet corn population and the maize 282 association panel (excluding the sweet corn lines within the maize 282 association panel). b XP-CLR estimated by comparing the maize 282 association panel against the sweet corn population (purple), the shrunken2 subpopulation (orange), and the sugary1 subpopulation (dark blue).

The $F_{\mathrm{ST}}$ between the sweet corn population and the maize 282 association panel (excluding the sweet corn lines in it) was 0.12 , indicating a moderate level of differentiation. As expected, based on the Tajima's $D$ results, the regions around the sul and sh2 loci show evidence of selection in sweet corn populations but not in maize 282 association panel varieties (Fig. 5a). In addition, distinct regions were found to have undergone selective sweeps on chromosomes 1,4 , and 5 . Although the Tajima's $D$ at these loci suggest selection is at play, determining the causal genes can be difficult due to the size of the loci.

To detect selective sweeps, XP-CLR was calculated for $50 \mathrm{~kb}$ windows across the genome between the maize 282 association panel and the sweet corn population. The sweet corn population was also further sub divided into sul and sh2 populations and compared against the maize 282 association panel. Using this approach, we identified windows with high XP-CLR peaks that overlapped with deviations in Tajima's D. These windows included candidate genes known to be involved in traits that are characteristic of sweet corn, such as plant height, leaf angle, tassel and ear architecture, early flowering, endosperm composition, and tillering (Fig. 5). Specifically, we found regions containing the genes drooping leaf1 $(\mathrm{ZmDRL1}$; Zm00001d028216), frigida-like protein1 (ZmFRL1; Zm00001 d028173), nuclear pore complex protein 96 (ZmNUP96; Zm00001d031680), dwarf plant1 (dwarf1; Zm00001d039634), fasciated ears3 (fea3; Zm00001d040130), NOD26-like membrane intrinsic protein1 (nip1a; Zm00001d016237), FTinteracting protein1 (ZmFTIP1; Zm00001d036804), tassel sheath4 (tsh4; Zm00001d020941), and naked endosperm2 (nkd2; Zm00001d026113) in addition to sugary1 and shrunken2. The drooping leaf1 encodes a CRC-like transcription factor and is associated with canopy structure and leaf angle ${ }^{41}$. In Arabidopsis, FRIGIDA-LIKE PROTEIN1 (FRL1) is required for FRIGIDA-mediated upregulation of the FLOWERING LOCUS C (FLC). The frll mutant in Arabidopsis suppresses FRIGIDA-mediated late flowering and up-regulation of $F L C^{42}$. Dwarf1 locus encodes a gibberellin 3-oxidase, and the mutant leads to short stature plants ${ }^{43}$. Another XP-CLR window contains the fasciated ears 3 gene, a leucine-rich-repeat receptor, and variation in this locus can impact the thickness of tassels and kernel row number ${ }^{44}$. The presence of tillers in sweet corn plants, and large tassel architecture could in part be controlled by the candidate genes nip $1 a^{45}$, and tassel sheath $4^{46}$, respectively. Two candidates for early flowering were also found, FT-interacting protein $1^{47}$ and nuclear pore complex protein $96^{48}$. A peak on chromosome 10 overlaps with

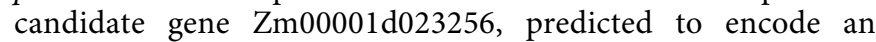
ADP glucose pyrophosphorylase small subunit. Finally, one of the regions under selection includes the candidate gene naked endosperm 2. The mutant for this locus produces multiple aleurone outer cell layers, affects starch content and composition as well as impacting greater than $6 \%$ of the transcriptome in these endosperm cell types ${ }^{49,50}$.

\section{Discussion}

Neolithic people domesticated maize from teosinte about 9000 years before present (yr B.P.), most likely in southwestern Mexico and then maize was carried by early agriculturalists throughout the Americas. Archeological evidence indicates that maize was being cultivated in what is now the southwestern United States about 4000 yr B.P ${ }^{51-54}$. By 1000 yr B.P., maize was already widely cultivated and a staple in the diet of the Eastern Woodlands people ${ }^{55}$. It was then rapidly adopted by many the people of eastern North America ${ }^{56,57}$. During this time, sweet corn was grown and maintained by indigenous peoples ${ }^{39,58-61}$, with modern sweet corn found to be related to Puebloan landraces grown by Native Americans in what is now the southwestern United States. Mutant sul alleles have been fixed and deliberately cultivated at least three different times in North America. The presence of three of the alleles in a conserved haplotype suggests a potential common origin. The su1 haplotype also seems to generally coincide with the origin of 'Northern Flints' in North America. It is unclear why this haplotype would have been maintained, although low recombination rates in this genetic background could explain this observation, which is supported by a large region with very low genetic diversity surrounding the sul locus on chromosome 4 (Fig. 5).

The shrunken 2 mutation is thought to be more recent, with records dating back to a maize stock from professor E.B. Mains created in $1943^{62}$. Previous work studying the sh2 gene documented the presence of a large insertion containing a Helitron and a complex rearrangement that caused the phenotype ${ }^{25}$. Our sweet corn genome provides a complete and consensus sh2- $R$ allele sequence, which highlights the role and importance of structural rearrangements as a mechanism to create genetic diversity in maize. 
Sweet corn is an important starchy vegetable with specific breeding targets, which have created bottlenecked populations that are very distinct from field corn. As an example, we see little overlap in our results with genomic regions under selection in modern field corn, recently presented and discussed in Wang et al. ${ }^{63}$. The generation of specialized genomic resources for sweet corn can not only provide support for future marker-based breeding decisions but also contribute towards the characterization of genes potentially not variable in field corn. Advances in next-generation sequencing and physical mapping technologies have reduced costs to enable routine sequencing of maize genomes by combining multiple technologies. Here, we present a highly contiguous genome assembly for a sweet corn inbred line, Ia453-sh2, to complement other field corn genome assemblies and provide a resource for comparative genomics available for the sweet corn community. Furthermore, population genetic analysis provided a list of candidate regions that have been differentially selected in sweet corn and field corn. These regions reflect the differential breeding choices and breeding targets that shaped modern sweet corn.

\section{Methods}

Plant material. The sweet corn (Z. mays) inbred line Ia453 with sh2-R allele (Ia453-sh2) was sequenced. The maize plants utilized for Illumina sequencing were grown in the greenhouse complex at the University of Florida (Gainesville, FL) in November of 2018. Young leaves from 3-week-old plants were harvested and frozen in liquid nitrogen. Genomic DNA was extracted using a modified CTAB method $^{64}$ with an RNase treatment carried out for $30 \mathrm{~min}$. DNA from 1-week old etiolated seedings from the same seed source was extracted utilizing the same method for PacBio sequencing.

PacBio and Illumina data generation. Large insert $(20 \mathrm{~kb})$ SMRTbell libraries were prepared and sequenced by the ICBR at the University of Florida using a PacBio SEQUEL system according to the recommended protocol (P/N 100-286000 Version 10 January 2018) with a few modifications. Briefly, high-integrity genomic DNA was further cleaned using the MoBio PowerClean DNA Cleanup Kit (\# 12877-50) kit. DNA was concentrated using AMPure beads (1:1 bead:sample ratio) and used for the subsequent SMRTbell library construction steps. The library construction steps included: ExoVII treatment, DNA Damage Repair, End Repair, Blunt-end ligation of SMRT bell adapters, and ExoIII/ExoVII treatment. The final library was size-selected in the SageELF ${ }^{\mathrm{TM}}$ instrument (Cat\# ELD 7510), using $0.75 \%$ agarose gel cassettes and the $1-18 \mathrm{~kb} v 2$ cassette definition program. The desired SageELF fractions were cleaned using AMPure magnetic beads (0.6:1.0 beads to sample ratio) and eluted in $15 \mu \mathrm{L}$ of $10 \mathrm{nM}$ Tris $\mathrm{HCl}, \mathrm{pH}$ 8.0. Between 6 and $8 \mathrm{pM}$ of library was loaded onto the PacBio SEQUEL sample plate for sequencing, using diffusion loading and $20 \mathrm{~h}$ movies (sequencing chemistry v3.0, SMRT Link 7.0). DNA extracted from the same sample was used to build standard 300-bp Illumina libraries. All Illumina libraries were prepared and sequenced with $150 \mathrm{bp}$ paired-end reads on an Illumina Hiseq 2500 system at GENEWIZ Inc. (South Plainfield, NJ).

PacBio long-read de novo assembly. Around 19.9 million PacBio SMRT SMRT subreads were error-corrected and assembled using Canu v1.8 ${ }^{13}$. For the full data set, only reads longer than $5 \mathrm{~kb}$ were corrected using the parameter: minReadLength $=5000$. By default, Canu only selects the longest $40 \times$ for correction. In order to get more corrected reads, the following parameter corOutCoverage $=60$ was used and resulted in $44.54 \times$ corrected sequence. The read trimming and unitig construction were run with the default parameters. To further improve the accuracy of the reference assembly, arrow (https://github.com/PacificBiosciences/ GenomicConsensus/) was used to correct the sequencing errors with default parameters.

BioNano mapping construction and hybrid assembly. Ultra-high molecular weight DNA was isolated from leaf tissues using the BioNano Prep Plant Tissue DNA Isolation Kit. The DNA was then labeled using the BioNano Direct Label and Stain method according to a protocol developed by Bionano Genomics ${ }^{65}$. The BioNano Saphyr system was used to stretch, separate and image the labeled DNA molecules. The resulting BNX file with the raw digitized molecules was filtered to include only molecules longer than $250 \mathrm{~kb}$ with at least nine fluorescent labels. The filtered molecule data set had a molecule N50 of $383 \mathrm{Mb}$ and was de novo assembled using the BioNano Solve 3.2 software. Maps were recursively refined and extended to construct the consensus maps. The total length of the final genome maps was $2.15 \mathrm{~Gb}$, with a map N50 of $119.5 \mathrm{Mb}$ and contained 69 BioNano genome maps. The PacBio contigs were in silico digested into consensus physical maps
(CMAPs) and were compared with the genome maps for hybrid scaffolding using the BioNano Solve software. The scaffolding was visualized and curated with the BioNano Access software. When conflicts occurred, the contigs were edited in accordance of alignment between PacBio contigs and the BioNano genome map. A total of 6890 PacBio contigs were linked into 29 super-scaffolds with an N50 of $120.95 \mathrm{Mb}$ and a total length of $2.14 \mathrm{~Gb}$. There were 8660 unscaffolded PacBio contigs. The total length of the super-scaffolds and unscaffolded PacBio contigs was $2.32 \mathrm{~Gb}$ with an $\mathrm{N} 50$ of $120.95 \mathrm{Mb}$.

There were 4734 overlaps between the scaffolds from the hybrid assembly with BioNano. When two PacBio contigs are found to share sequences in their extremes, the software will output $13-\mathrm{N}$ gaps between two contigs. Flanking $100 \mathrm{~kb}$ sequences around the 13-N gaps were extracted and merged by Minimus 2 when they overlapped $^{66}$. A total of 4316 scaffolds were successfully merged. A python-based command line tool reform (https://github.com/gencorefacility/reform) was used to put back the merged sequences into the hybrid assembly.

Hi-C library preparation and sequencing. A Hi-C library (Dovetail Genomics LLC, Santa Cruz, CA) was generated using the DpnII restriction endonuclease (GATC). Briefly, this entailed reconstituting chromatin using purified histones and chromatin assembly factors, followed by cross-linking the chromatin using formaldehyde. DNA was then digested using DpnII restriction enzymes, $5^{\prime}$ overhangs filled in with biotinylated nucleotides and free blunt ends were ligated. After ligation, cross-links are reversed and the DNA was purified from protein. The DNA was then sheared to a mean fragment size of about $350 \mathrm{bp}$, and biotincontaining fragments were isolated using streptavidin beads. The resulting DNA fragments were taken through a standard Illumina library preparation and sequenced on HiSeq X-Ten $(2 \times 150$ bp paired-end reads) to provide $\sim 56 \times$ physical genome coverage.

Pseudomolecule construction with HiRise. The Dovetail Hi-C library were used to scaffold the 29 super-scaffolds and 8660 unscaffoled PacBio contigs through Dovetail's HiRise pipepline. Dovetail Hi-C library sequences were aligned back to the input de novo assembly using a modified version of SNAP (http://snap.cs. berkeley.edu/). The read pairs that mapped uniquely on the assembly were used to generate a likelihood model to estimate the genomic distance between read pairs, identify and break the putative misjoins, score the prospective joins and make joins above a selected threshold. There were no breaks and 12 joins made by HiRise. Dovetail HiRise assembly contains 10 chromosomes and 8440 unscaffolded contigs (Supplementary Table 7).

To further polish the pseudomolecules, a $\sim 23 \times$ coverage of paired-end Illumina whole genome sequencing library was generated for sequencing polishing using ntEdit ${ }^{15}$. The ntHits was first ran with parameters "-k $25-\mathrm{c} 2$ " to build a Bloom filter, which is read by ntEdit to polish the assembly with default parameters. A total of 832,323 changes were corrected, including $31.29 \%$ SNPs and $68.7 \%$ small indels (2-25 bps).

Genome size estimation. The genome size was estimated using k-mer histograms computed from the error-corrected PacBio reads using the program jellyfish v.2.3.067, with word sizes $(k)$ of 25 . Furthermore, four genotypes were used to estimate genome size using flow cytometry. The B73 genotype was used as the reference standard and we estimated the genome size for Ia453-sh2, F7 and W22. Flow cytometry measurements were made on five biological replicates per genotype, and the genome size estimated as a ratio relative to B73 was reported as the average across the five replicates (Supplementary Table 2). Sample preparation: five seeds per genotype were imbibed for $48 \mathrm{~h}$ in full darkness, and the embryo was dissected and used for the analysis following the methodology described by Rios et al. ${ }^{68}$. Specifically, the embryo was mixed with $500 \mu \mathrm{l}$ of extraction buffer (CyStain PI absolute P, Partec $\mathrm{GmbH}$, Münster) and chopped using a sharp razor blade for $30 \mathrm{~s}$ in a petri dish kept on ice. The excised tissue was then incubated on ice for another $30 \mathrm{~s}$. Samples were then filtered using Partec $50 \mu \mathrm{m}$ CellTrics (Partec $\mathrm{GmbH}$, Münster), and stained using $2 \mathrm{ml}$ of staining solution containing propidium iodide and RNase (CyStain PI absolute P, Partec GmbH, Münster). Samples were incubated on ice for at least $30 \mathrm{~min}$ and analyzed immediately with the BD Accuri C6 Flow Cytometer with laser illumination at $488 \mathrm{~nm}$ and a 610/20 nm filter with the FL2 detector (Accuri Cytometers, Ann Arbor, MI) at the University of Florida Interdisciplinary Center for Biotechnology Research, Gainesville, FL. The gating strategy is provided in the Supplementary Fig. 9. For each sample at least 5000 nuclei were counted and analyzed using the BD Accuri CFlow software (Version 1.0.264; BD Biosciences, CA, USA), and only samples whose G1 peak had a coefficient of variation (CV FL2-A) smaller than $10 \%$ were considered for analysis. The amount of DNA contained within diploid nuclei (2C-DNA content) for Ia453-sh2, F7 and W22 was calculated based on the fluorescence intensity recorded for their G1 peaks and compared to the G1 peak of the reference standard (B73).

Assembly evaluation. The genome completeness from contig to chromosomelevel assembly was assessed using the benchmarking universal single-copy orthologs (BUSCO) v3.0217. The final assembly was tested against the Plantae BUSCO "Embryophyta_odb9" database, which contained 1440 protein sequences and orthogroup annotations for major clades. This result was compared with that of 
B73, Mo17, W22, EP1, F7, and DK105 genomes. The assembly of line PH207 was not included due to lower assembly quality. The LAI-a method to evaluate genome assembly completeness based on the quality of the assembly of repeat sequences-was also run for all the genomes above using the LTR_retriever pipeline ${ }^{16}$

PacBio Iso-seq library preparation, sequencing, and assembly. Total RNA was extracted from leaf, stem, silk, husk, ear and pollen tissues using Trizol and RNeasy MinElute (Qiagen) RNA clean up kit, with an Dnase I treatment of $20 \min ^{69}$. The RNA integrity was assessed with a Bioanalyzer prior to the construction of the IsoSeq library. The Iso-Seq libraries were prepared and sequenced by Interdisciplinary Center for Biotechnology Research (ICBR) at the University of Florida using a PacBio Sequel system. PacBio Iso-Seq data were analyzed by running the IsoSeq3 v3.1 in PacBio SMART Analysis v7.0 (https://github.com/PacificBiosciences/ IsoSeq3) to generate high-quality, full-length transcript sequences.

RNA-seq and transcriptome assembly. RNA-seq data was also generated for endosperm sampled 14 days after pollination. The total RNA was extracted using RNeasy MinElute (Qiagen) following the manufacturer's recommended protocol. The total RNA was processed using the TruSeq RNA Sample Preparation kit followed by sequencing on the Illumina HiSeq 2500 platform. The software Trimmomatic $\mathrm{v} 0.36$ was used to trim adapter sequences of RNA sequencing reads ${ }^{70}$. The paired-end reads were merged using PEAR v0.9.6 ${ }^{71}$, which were used for following transcriptome assembly. The de novo transcriptome assembly was performed using Trinity v2.8.4 with default parameters ${ }^{72}$. The genome-guided transcriptome assembly was performed with HISAT2 v2.1.0 73,74 and StringTie v1.3. $4^{75}$. The genome index was built using HISAT2-build and the clean transcriptome reads were mapped to the sweet corn genome using HISAT2. The genome-guided transcriptome assembly was performed using StringTie. The resulting StringTie and Trinity assemblies were supplied to PASA v2.2.076 in order to build comprehensive transcriptome database.

Protein-coding gene annotation. MAKER-P v2.31.10 22 was used to annotate genes in the Ia453-sh2 genome with the evidence data from the annotation of the B73v4 genome and some additional evidence as outlined below. RepeatMasker was used to mask low complexity genomic sequence using exemplar transposon sequences ${ }^{77}$. The annotated proteins from Sorghum bicolor, Oryza sativa, Setaria italica, Brachypodium distachyon, and Arabidopsis thaliana, downloaded from Gramene.org release $48^{78}$, were used for protein evidence. A set of 69,163 publicly available full-length cDNAs and 2,019,896 publicly available ESTs deposited in GeneBank $^{79}, 1,574,442$ Trinity-assembled transcripts from 94 B73 RNA-Seq experiments ${ }^{80}, 112,963$ transcripts assembled from deep sequencing of a B73 seedling ${ }^{81}$ and 111,151 high quality transcripts from B73 Iso-seq ${ }^{82}$ were used as transcript evidence. In addition, the following evidences were included: 75,945 PASA transcripts from Ia453-sh2 endosperm, 79,855 full-length transcripts from Ia453-sh2 Iso-seq from multiple tissues, 143,679 transcripts and proteins from B73v4 annotation ${ }^{18}$, 36,507 transcripts and proteins from CML247 draft genome annotation $^{83}, 48,140$ transcripts and proteins from DK105 genome annotation ${ }^{21}$, 46,105 transcripts and proteins from EP1 genome annotation ${ }^{21}, 48,370$ transcripts and proteins from F7 genome annotation ${ }^{21}, 46,530$ transcripts and proteins from Mo17 genome annotation ${ }^{20}$, 40,557 transcripts and proteins from PH207 genome annotation $^{84}$, and 51,716 transcripts and proteins from W22 genome annotation ${ }^{19}$. For gene prediction, AUGUSTUS ${ }^{23}$ and FGENESH ${ }^{24}$ were trained on "maize5" and "monocots" models. The working gene set $(47,168$ genes and 86,182 transcripts) was identified in Ia453-sh2 genome. All predicted proteins were annotated using InterProScan (version 5.35-74.0) and running BLASTP against UniProt database. Predicted genes were filtered according to annotation evidence distance scores (AED) calculated by MAKER-P and filtered to avoid overlapped with repeat-masked regions. In the end, 38,384 high-confidence protein-coding genes and 72,762 transcripts remained as a final set (Supplementary Fig. 3).

TE annotation. TEs were identified in the sweet corn inbred line Ia453-sh2 and six field corn genome assemblies (B73, Mo17, W22, EP1, F7, and DK105) using independent de novo prediction tools: EDTA v1.8.5 ${ }^{85}$, TARGeT ${ }^{86}$, and SINEFinder ${ }^{87}$.

Class I (retrotransposons). LTR. LTR retrotransposons elements were predicted and annotated using EDTA package ${ }^{85}$. EDTA utilized a combination of LTR_FIN$\mathrm{DER}^{88}$ and LTR harvest ${ }^{89}$ with LTR retriever to perform whole-genome LTR retrotransposons annotations. The redundant sequences, nested insertions and protein-coding sequences were removed by EDTA in the final non-redundant TE libraries. Intact LTR retrotransposons were identified and the insertion time of those intact LTR retrotransposons were estimated using LTR retriever ${ }^{90}$.

LINE. Long interspersed nuclear elements (LINEs) were identified using $\mathrm{TARGeT}^{86}$ similarity searches on the maize TE consortium (MTEC) database ${ }^{77,91}$, as described in detail for $\mathrm{B} 73 \mathrm{v} 4^{18}$ genome annotation.

SINE. Short interspersed nuclear elements (SINEs) were annotated using SINEFinder ${ }^{87}$ with default parameters (-T chunkwise -V1).
Class II (DNA transposons). TIR. TIR-learner ${ }^{92}$ was used by $\mathrm{EDTA}^{85}$ to predict and annotate TIR transposons.

Helitron. HelitronScanner ${ }^{93}$ was used by EDTA ${ }^{85}$ to predict and annotate Helitron elements.

Detection of SNPs, small indels, and structural variations. SNPs and small indels (length $<\mathrm{Xbp}$ ) were identified between Ia453-sh2 and the other six field corn genomes using MUMmer v3.23 ${ }^{94}$. First, nucmer from the MUMmer was used to generate the alignment with parameters "-mum -g $1000-\mathrm{c} 90-\mathrm{l} 40$ ". Then the alignment files were filtered to generate 1-to-1 mapping by delta-filter with parameters "- $r-q$ ". The SNPs and small indels were called from 1-to-1 alignment blocks by show-snp with parameter "-ClrTH". The output of NUCmer was also analyzed using Assemblytics ${ }^{55}$, a Web-based SV analytic tool, to identify the large structural variations $(100-100,000 \mathrm{bp})$.

OrthoMCL analysis. Orthologous gene clusters were assigned for Ia453-sh2 and six field corn genomes using OrthoMCL ${ }^{26}$ with the default parameters. Splice variants and incomplete gene models in the genomes were removed, and an all-byall comparison was then performed using BLASTP with an $E$ value of $1 \times 10^{-5}$. A total of 350,089 protein sequences were clustered into 45,600 gene families.

Identification of la453-sh2-specific sequences, clusters, and genes. Ia453-sh2 specific sequences were detected by first dividing the genome into sliding windows of length $1000 \mathrm{bp}$ with a $500 \mathrm{bp}$ overlap. Then, those small windows were aligned to the other six field corn genomes using BWA-MEM ${ }^{96}$ (v0.7.17) with parameters (-w $500--\mathrm{M})$. If the sequences of windows failed to be aligned or had less than $20 \%$ of the sequences aligned to the six field corn genomes but could be perfectly aligned to the Ia453-sh2 genome, those sequences are defined as Ia453-sh2-specific sequences.

The Ia453-sh2-specific genes were initially identified if the gene had more than $80 \%$ of CDS sequences overlapped with Ia453-sh 2 specific sequences. A second method was used to remove potential false positives. The Ia453-sh2 CDS were aligned to the six field corn genomes using GMAP (gmap-2019-06-10) to calculate the query coverage of alignment and alignment identity ${ }^{97}$. The parameters query coverage of alignment and alignment identity were used to categorize the Ia453-sh2 genes into highly conserved genes with $100 \%$ query coverage and $100 \%$ identity; genes with mutations with $100 \%$ coverage and $90-100 \%$ identity; and genes with structural variations with $50-100 \%$ coverage and $90-100 \%$ identity (Supplementary Fig. 5). The remaining genes that failed to be aligned, that had the query coverage of alignment less than $50 \%$, or that had alignment identity less than $90 \%$ were defined as Ia453-sh2 specific genes. The final set of Ia453-sh2 specific genes was defined if they were detected by both methods.

Paired-end RNA-Seq reads from endosperm were aligned against Ia453-sh2 genome using STAR v2.7.3 ${ }^{98}$ to check the gene expression of the identified Ia453sh2 specific genes. Publicly available $3^{\prime}$ RNAseq data from seven tissues of five sweet corn lines (P39, IL14H, Il677a, Il101T, and IA2132) and five field corn lines (B73, Mo17, W22, EP1, and F7) were used the check the gene expression of Ia453sh2-specific genes in different tissues ${ }^{32}$.

Structural variations of $\boldsymbol{s h 2}-\boldsymbol{R}$ allele. The Sh2 gene sequence from B73 was aligned against Ia453-sh2 genome using BLAST and MUMmer v3.23 $3^{94}$ to compare the structural variations between Sh2 from B73 and sh2-R allele in Ia453-sh2. Paired-end RNA-seq reads from endosperm were aligned against Ia453-sh2 genome using STAR v2.7.3a ${ }^{98}$ to verify the gene expression of two predicted genes (Zm00045a021195 and Zm00045a021196) in the Ia453 sh2-R region.

Gene ontology enrichment analyses. The Bioconductor package topGO (version 2.32.0) was used for the gene ontology enrichment analysis with all annotated genes as the universe set. In the topGO analysis, GO terms significance of interest were assessed based on Fisher's exact test statistic using 0.05 as the significance threshold.

Population genetic and phylogenetic analyses. We created a genotyping-bysequencing build using publicly available sequences comprising 5318 diverse maize accessions were compiled from recent studies $57,99,100$. This set includes a total of 822 sweet corn line, 340 teosinte and a diverse set of tropical and temperateadapted non-sweet lines. The TASSEL 5 GBS pipeline ${ }^{101}$ was used to call SNPs from all the sequencing data using the maize reference genome B73 v4, and a minimum minor allele cutoff of 10 observations, resulting in 859,632 SNPs in total. This SNP set was used for the calculation of Tajima's $D$ and XP-CLR described below. For the phylogenetic analysis, the SNPs were filtered using VCFtools ${ }^{102}$. SNPs were filtered to have a minor allele frequency $>0.1$, a linkage disequilibrium $r^{2}<0.2$, and a percentage of missing data per SNP $<30 \%$, resulting in a reduced set of 9725 SNPs.

A maximum likelihood-based approach was used to construct a phylogenetic tree. Maximum likelihood phylogenies were inferred with RAxML-NG ${ }^{103}$. Five parsimony trees and five random trees were generated to initialize the tree searches. For all ten trees, the GTR $+\mathrm{G}$ model was employed and an LH epsilon of 10 was chosen. The phylogenetic tree with the lowest final log likelihood at the conclusion 
of the tree search was chosen. The final phylogenetic tree was plotted in R with the ggplot2 and ggtree packages ${ }^{104,105}$. Genotype classifications were assigned and colored according to Supplementary Data 4. The same SNP set was also used to calculate principal component analysis and DAPC in the R package adegenet ${ }^{106}$

Fixation index and population structure analyses. To determine the level of genetic differentiation among different populations of maize, $F_{\mathrm{ST}}$ was calculated as described by Weir and Cockerham ${ }^{107}$. VCFtools was used to calculate the $F_{\mathrm{ST}}$ of every SNP between sweet corn and each other population of maize. A random set of 10,000 SNPs was used for population structure analysis. The Bayesian clustering method implemented in STRUCTURE, v.2.3.4 was used to identify clusters of genetically related individuals ${ }^{108}$. Ten independent replications were performed for each of $K=$ $1-16$ ( $K=$ number of genetic clusters) with a burn-in of 10,000 and 20,000 iterations. The online version of STRUCTURE HARVESTER ${ }^{109}$ was used to determine the optimal K based on the Evanno method ${ }^{110}$ (eight was the selected optimal).

Population analysis of the sugary1 gene. For su1 haplotype heatmap, fasta sequences of the sugaryl locus in 1208 genotypes were obtained by updating the B73 sequence with SNPs identified on HapMap3. A distance matrix was then calculated using the dist.dna() function from the R package phangorn ${ }^{111}$ with the total number of SNPs between each pair of genotypes as the distance. A corresponding heatmap of the distance matrix was then plotted using ggplot2.

For sul tree, a neighbor joining tree of the $s u 1$ locus was calculated using a representative subset of 51 genotypes. The JC69 nucleotide substitution model was chosen. The tree was then rooted with the sugaryl from Tripsacum dactyloides and plotted using the R packages ggplot2 and ggtree.

For sul multiple alignment, the sul locus sequence was determined from twelve overlapping PCR fragments, amplified from genomic DNA, spanning $\sim 8.4 \mathrm{~kb}$ including all of the transcribed region and parts of the $5^{\prime}$ - and $3^{\prime}$-UTRs. The locus was then sequenced from five introgressions of the A632 inbred background, each homozygous for an independent sul- mutation identified in a survey of extant sweet corn lines ${ }^{33}$. The su1-nc and su1-sw alleles are nearly identical to su1-ne (su1-Ref) with the exceptions of causative agent SNPs, specifically R504C in su1-nc and N561S in su1$s w$, and one additional SNP in sul-nc compared to sul-Ref. In contrast, sul-cm and su1-pu were nearly identical to the B73 allele and lacked all SNPs identified in su1-Ref.

These results revealed two distinct sul haplotype groups in extant maize lines derived independently from long-divergent progenitor alleles. The group containing sul-Ref was designated haplotype group 1 and that containing the B73 allele as haplotype group 2. A multiple alignment of the sul locus was constructed on a subset of 9 genotypes including teosinte, dent corn, and the different sulmutant alleles. For B73, Mo17, and PI566673, sequences were extracted from genome assemblies published at MaizeGDB. Lastly, the TIL genotypes were retrieved by updating the B73 sequence with HapMap3 SNPs.

Identification of selection sweeps. To identify loci deviating from the mutationdrift equilibrium, we used 859,632 SNPs and calculated Tajima's $D$ for $10 \mathrm{~kb}$ sliding windows across the genome for the maize 282 association panel and sweet corn (along with its sugary1 and shrunken2 subsets). Tajima's $D$ was calculated using the $\mathrm{R}$ package PopGenome ${ }^{112}$. A locally estimated scatterplot smoothing (LOESS) was applied to the Tajima's $D$ values from each population and subsequently plotted with ggbio ${ }^{113}$.

To detect selective sweeps between the maize 282 association panel and sweet corn populations (along with its sugary1 and shrunken2 subsets), the crosspopulation composite likelihood ratio (XP-CLR) test was employed ${ }^{114}$. We used the sweet corn as a reference and the maize 282 association panel as a query to identify the selection sweeps. The selection sweeps were scanned with a sliding window of $50 \mathrm{~kb}$ and a step of $100 \mathrm{bp}$. The maximum number of SNPs in each window was set up as 50 and the correlation levels were set up as 0.7 . XP-CLR values from the three comparisons were then plotted with ggbio ${ }^{113}$. The regions with XP-CLR values in the top $1 \%$ of the empirical distributions were designated as candidate sweeps and the candidate genes were identified within the selected candidate genomic regions. We note that other genes within the selected candidate regions may actually be the ones originally associated with the selective sweep, and future research will be required to validate and establish the functional link between the causal gene and the sweep.

Reporting summary. Further information on research design is available in the Nature Research Reporting Summary linked to this article.

\section{Data availability}

Data supporting the findings of this work are available within the paper and its Supplementary Information files. A reporting summary for this Article is available as a Supplementary Information file. All other raw data are available from the corresponding author upon request. The genome assembly have been deposited in NCBI database under BioProject accession PRJNA646414, BioSample accession SAMN15543012, and GeneBank accession JACHTI000000000.1/. RNA-seq data have been deposited in NCBI Sequence Read Archive under accession SRR12300193, SRR12300194, SRR12300195, and SRR12300196, as well as in BioProject under accession PRJNA647770. The protein coding gene, transposon annotations, and the VCF file of 5381 maize lines are publicly available at CyVerse. Source data are provided with this paper.

Received: 10 July 2020; Accepted: 26 January 2021; Published online: 23 February 2021

\section{References}

1. Lertrat, K. \& Pulam, T. Breeding for increased sweetness in sweet corn. Int. J. Plant Breed. 1, 27-30 (2007).

2. USDA-NASS. United States Department of Agriculture-National Agricultural Statistics Service QuickStats. (2019).

3. USDA/HHS. The Report of the Dietary Guidelines Advisory Committee on the Dietary Guidelines for Americans, 2015, to the Secretary of Agriculture and the Secretary of Health and Human Services. (2015).

4. Rahman, A., Wong, K., Jane, J., Myers, A. M. \& James, M. G. Characterization of SU1 isoamylase, a determinant of storage starch structure in maize. Plant Physiol. 117, 425-435 (1998).

5. Tsai, C. Y. \& Nelson, O. E. Starch-deficient maize mutant lacking adenosine dephosphate glucose pyrophosphorylase activity. Science 151, 341-343 (1966).

6. Creech, R. G. Genetic control of carbohydrate synthesis in maize endosperm. Genetics 52, 1175-1186 (1965).

7. Tracy, W. F. History genetics and bredding of supersweet (shrunken2) sweet corn. Plant Breed. Rev. 14, 189-236 (1997).

8. Boyer, C. D. \& Shannon, J. C. The use of endosperm genes for sweet corn improvement. Plant Breed. Rev. 1, 193-161 (1984).

9. Marshall, S. W. \& Tracy, W. F. Sweet corn. In (eds. Ramstad, P. E. \& White, P.) Corn Chemistry and Technology. 537-569 (Minneapolis, MN, 2003).

10. Hannah, L. C. \& Nelson, O. E. Characterization of ADP-glucose pyrophosphorylase from shrunken-2 and brittle-2 mutants of maize. Biochem. Genet. 14, 547-560 (1976).

11. Tracy, W. F., Shuler, S. L. \& Dodson-Swenson, H. The use of endosperm genes for sweet corn improvement: a review of developments in endosperm genes in sweet corn since the seminal publication in Plant Breeding Reviews, Volume 1, by Charles Boyer and Jack Shannon (1984). Plant Breed. Rev. 43, 215-241 (2019).

12. $\mathrm{Xu}, \mathrm{G}$. et al. Complex genetic architecture underlies maize tasse domestication. N. Phytol. 214, 852-864 (2017).

13. Koren, $\mathrm{S}$. et al. Canu: scalable and accurate long-read assembly via adaptive $\mathrm{k}$ mer weighting and repeat separation. Genome Res. 27, 722-736 (2017).

14. Putnam, N. et al. Chromosome-scale shotgun assembly using an in vitro method for long-range linkage. Genome Res. 26, 342-350 (2016).

15. Warren, R. L. et al. ntEdit: scalable genome sequence polishing. Bioinformatics 35, 4430-4432 (2019).

16. Ou, S., Chen, J. \& Jiang, N. Assessing genome assembly quality using the LTR assembly index (LAI). Nucleic Acids Res. 46, e126 (2018).

17. Simão, F. A., Waterhouse, R. M., Ioannidis, P., Kriventseva, E. V. \& Zdobnov, E. M. BUSCO: assessing genome assembly and annotation completeness with single-copy orthologs. Bioinformatics 31, 3210-3212 (2015).

18. Jiao, Y. et al. Improved maize reference genome with single-molecule technologies. Nature 546, 524-527 (2017).

19. Springer, N. M. et al. The maize W22 genome provides a foundation for functional genomics and transposon biology. Nat. Genet. 50, 1282-1288 (2018).

20. Sun, S. et al. Extensive intraspecific gene order and gene structural variations between Mol7 and other maize genomes. Nat. Genet. 50, 1289-1295 (2018).

21. Haberer, G. et al. European maize genomes unveil pan-genomic dynamics of repeats and genes. bioRxiv https://doi.org/10.1101/766444 (2019).

22. Campbell, M. S. et al. MAKER-P: a tool kit for the rapid creation, management, and quality control of plant genome annotations. Plant Physiol. 164, 513-524 (2014).

23. Stanke, M. et al. AUGUSTUS: ab initio prediction of alternative transcripts Nucleic Acids Res. 34, W435-W439 (2006).

24. Salamov, A. A. \& Solovyev, V. V. Ab initio gene finding in Drosophila genomic DNA. Genome Res. 10, 516-522 (2000).

25. Kramer, V., Shaw, J. R., Senior, M. L. \& Hannah, L. C. The sh2-R allele of the maize shrunken-2 locus was caused by a complex chromosomal rearrangement. Theor. Appl. Genet. 128, 445-452 (2015).

26. Li, L., Stoeckert, C. J. \& Roos, D. S. OrthoMCL: identification of ortholog groups for eukaryotic genomes. Genome Res. 13, 2178-2189 (2003).

27. Gipson, A. B. et al. Disruptions in valine degradation affect seed development and germination in Arabidopsis. Plant J. 90, 1029-1039 (2017).

28. Cheng, Y. T. et al. Stability of plant immune-receptor resistance proteins is controlled by SKP1-CULLIN1-F-box (SCF)-mediated protein degradation. Proc. Natl Acad. Sci. USA 108, 14694-14699 (2011).

29. Gou, $\mathrm{M}$. et al. The F-box protein CPR1/CPR30 negatively regulates $\mathrm{R}$ protein SNC1 accumulation. Plant J. 69, 411-420 (2012). 
30. Dielen, A.-S. et al. The $20 \mathrm{~S}$ proteasome a 5 subunit of Arabidopsis thaliana carries an RNAse activity and interacts in planta with the Lettuce mosaic potyvirus HcPro protein. Mol. Plant Pathol. 12, 137-150 (2011).

31. Guo, L. et al. Connections between sphingosine kinase and phospholipase D in the abscisic acid signaling pathway in Arabidopsis. J. Biol. Chem. 287, 8286-8296 (2012).

32. Kremling, K. A. et al. Dysregulation of expression correlates with rare-allele burden and fitness loss in maize. Nature 555, 520-523 (2018).

33. Tracy, W. F., Whitt, S. R. \& Buckler, E. S. Recurrent mutation and genome evolution: example of Sugaryl and the origin of sweet maize. Crop Sci. 46, S49-S-54 (2006).

34. Carey, E. E., Dickinson, D. B. \& Rhodes, A. M. Sugar characteristics of sweet corn populations from a sugary enhancer breeding program. Euphytica 33, 609-622 (1984).

35. Zhang, X. et al. Maize sugary enhancer1 (se1) is a gene affecting endosperm starch metabolism. Proc. Natl Acad. Sci. USA 116, $20776-20785$ (2019).

36. Dinges, J. R., Colleoni, C., Myers, A. M. \& James, M. G. Molecular structure of three mutations at the maize sugary 1 locus and their allele-specific phenotypic effects. Plant Physiol. 125, 1406-1418 (2001).

37. Tracy, W. F. Vegetable uses of maize (corn) in pre-columbian America. HortScience 34, 812-813 (1999).

38. Bukowski, R. et al. Construction of the third-generation Zea mays haplotype map. Gigascience 7, 1-12 (2018)

39. Wellhausen, E. J., Roberts, L. M. \& Efrain Hernandez X. Races of Maize in Mexico. (Bussey Institute, Harvard University, Cambridge, MA, 1952).

40. Zhang, $X$. et al. The tin 1 gene retains the function of promoting tillering in maize. Nat. Commun. 10, 5608 (2019).

41. Strable, J. et al. Maize $Y A B B Y$ genes drooping leaf1 and drooping leaf2 regulate plant architecture. Plant Cell 29, 1622-1641 (2017).

42. Schläppi, M. R. FRIGIDA LIKE 2 is a functional allele in Landsberg erecta and compensates for a nonsense allele of FRIGIDA LIKE 1. Plant Physiol. 142, 1728-1738 (2006).

43. Chen, Y. et al. The maize DWARF1 encodes a gibberellin 3-oxidase and is dual localized to the nucleus and cytosol. Plant Physiol. 166, 2028-2039 (2014).

44. Je, B. I. et al. Signaling from maize organ primordia via FASCIATED EAR3 regulates stem cell proliferation and yield traits. Nat. Genet. 48, 785-791 (2016).

45. Bouchet, S. et al. Association mapping for phenology and plant architecture in maize shows higher power for developmental traits compared with growth influenced traits. Heredity 118, 249-259 (2017).

46. Chuck, G., Whipple, C., Jackson, D. \& Hake, S. The maize SBP-box transcription factor encoded by tasselsheath4 regulates bract development and the establishment of meristem boundaries. Development 137, 1243-1250 (2010).

47. Liu, L. et al. FTIP1 is an essential regulator required for florigen transport. PLoS Biol. 10, e1001313 (2012).

48. Cheng, Z. et al. Nup96 and HOS1 are mutually stabilized and gate CONSTANS protein level, conferring long-day photoperiodic flowering regulation in Arabidopsis. Plant Cell 32, 374-391 (2020).

49. Yi, G., Neelakandan, A. K., Gontarek, B. C., Vollbrecht, E. \& Becraft, P. W The naked endosperm genes encode duplicate INDETERMINATE domain transcription factors required for maize endosperm cell patterning and differentiation. Plant Physiol. 167, 443-456 (2015).

50. Gontarek, B. C., Neelakandan, A. K., Wu, H. \& Becraft, P. W. NKD transcription factors are central regulators of maize endosperm development. Plant Cell 28, 2916-2936 (2016).

51. Vierra, B. J. E. The Late Archaic across the Borderlands: From Foraging to Farming (University of Texas Press, 2005).

52. Gregory, D. A., Stevens, N. M., Nails, F. L., Schurr, M. R. \& Diehl, M. W. E. Excavations in the Santa Cruz River Floodplain: Further Investigations at Los Pozos (Center for Desert Archaeology, Tucson, 2007).

53. Wills, W. H. Early Prehistoric Agriculture in the American Southwest (School of American Research Press, Santa Fe, 1988).

54. da Fonseca, R. R. et al. The origin and evolution of maize in the Southwestern United States. Nat. Plants 1, 14003 (2015).

55. Emerson, T., Hedman, K., Simon, M., Fort, M. \& Witt, K. Isotopic confirmation of the timing and intensity of maize consumption in greater Cahokia. Am. Antiquity 85, 241-262 (2020).

56. Matsuoka, Y. et al. A single domestication for maize shown by multilocus microsatellite genotyping. Proc. Natl Acad. Sci. USA 99, 6080-6084 (2002).

57. Swarts, K. et al. Genomic estimation of complex traits reveals ancient maize adaptation to temperate North America. Science 357, 512-515 (2017).

58. Hendry, G. W. Archaeological evidence concerning the origin of sweet maize. J. Am. Soc. Agron. 22, 508-514 (1930).

59. Grobman, A., Salhuana, W. \& Sevilla, R. Races of Maize in Peru. (National Academy of Science, National Research Council, Pub. 915, Washington, D.C., 1961).

60. Carter, G. F. Sweet corn among the Indians. Geog. Rev. 38, 206-221 (1948)

61. Will, G. F. \& Hyde, G. E. Corn among the Indians of the Upper Missouri. (W. H. Miner, St. Louis, MO, 1917).
62. Mains, E. B. Heritable characters in maize; linkage of a factor for shrunken endosperm with the al factor for aleurone color. J. Hered. 40, 21-24 (1949)

63. Wang, B. et al. Genome-wide selection and genetic improvement during modern maize breeding. Nat. Genet. 52, 565-571 (2020).

64. Porebski, S., Bailey, L. G. \& Baum, B. R. Modification of a CTAB DNA extraction protocol for plants containing high polysaccharide and polyphenol components. Plant Mol. Biol. Rep. 15, 8-15 (1997).

65. Ou, S. et al. Effect of sequence depth and length in long-read assembly of the maize inbred NC358. Nat. Commun. 11, 2288 (2020).

66. Sommer, D. D., Delcher, A. L., Salzberg, S. L. \& Pop, M. Minimus: a fast, lightweight genome assembler. BMC Bioinform. 8, 64 (2007).

67. Marçais, G. \& Kingsford, C. A fast, lock-free approach for efficient parallel counting of occurrences of k-mers. Bioinformatics 27, 764-770 (2011).

68. Rios, E. F., Kenworthy, K. E. \& Munoz, P. R. Association of phenotypic traits with ploidy and genome size in annual ryegrass. Crop Sci. 55, 2078-2090 (2015).

69. Bihmidine, S., Baker, R. F., Hoffner, C. \& Braun, D. M. Sucrose accumulation in sweet sorghum stems occurs by apoplasmic phloem unloading and does not involve differential Sucrose transporter expression. BMC Plant Biol. 15, 186 (2015).

70. Bolger, A. M., Lohse, M. \& Usadel, B. Trimmomatic: a flexible trimmer for Illumina sequence data. Bioinformatics 30, 2114-2120 (2014).

71. Zhang, J., Kobert, K., Flouri, T. \& Stamatakis, A. PEAR: a fast and accurate Illumina Paired-End reAd mergeR. Bioinformatics 30, 614-620 (2014).

72. Grabherr, M. G. et al. Full-length transcriptome assembly from RNA-Seq data without a reference genome. Nat. Biotechnol. 29, 644-652 (2011).

73. Kim, D., Langmead, B. \& Salzberg, S. L. HISAT: a fast spliced aligner with low memory requirements. Nat. Methods 12, 357-360 (2015).

74. Pertea, M., Kim, D., Pertea, G. M., Leek, J. T. \& Salzberg, S. L. Transcript-level expression analysis of RNA-seq experiments with HISAT, StringTie and Ballgown. Nat. Protoc. 11, 1650-1667 (2016).

75. Pertea, M. et al. StringTie enables improved reconstruction of a transcriptome from RNA-seq reads. Nat. Biotechnol. 33, 290-295 (2015).

76. Haas, B. J. et al. Improving the Arabidopsis genome annotation using maximal transcript alignment assemblies. Nucleic Acids Res. 31, 5654-5666 (2003).

77. Schnable, P. S. et al. The B73 maize genome: complexity, diversity, and dynamics. Science 326, 1112-1115 (2009).

78. Tello-Ruiz, M. K. et al. Gramene 2016: comparative plant genomics and pathway resources. Nucleic Acids Res. 44, D1133-D1140 (2016).

79. Soderlund, C. et al. Sequencing, mapping, and analysis of 27,455 maize fulllength cDNAs. PLoS Genet. 5, e1000740 (2009).

80. Law, M. et al. Automated update, revision, and quality control of the maize genome annotations using MAKER-P improves the B73 RefGen_v3 gene models and identifies new genes. Plant Physiol. 167, 25-39 (2015).

81. Martin, J. A. et al. A near complete snapshot of the Zea mays seedling transcriptome revealed from ultra-deep sequencing. Sci. Rep. 4, 4519 (2014)

82. Wang, B. et al. Unveiling the complexity of the maize transcriptome by singlemolecule long-read sequencing. Nat. Commun. 7, 11708 (2016).

83. Lu, F. et al. High-resolution genetic mapping of maize pan-genome sequence anchors. Nat. Commun. 6, 6914 (2015).

84. Hirsch, C. N. et al. Draft assembly of elite inbred line $\mathrm{PH} 207$ provides insights into genomic and transcriptome diversity in maize. Plant Cell 28, 2700-2714 (2016).

85. Ou, S. et al. Benchmarking transposable element annotation methods for creation of a streamlined, comprehensive pipeline. Genome Biol. 20, 275 (2019).

86. Han, Y., Burnette, J. M. \& Wessler, S. R. TARGeT: a web-based pipeline for retrieving and characterizing gene and transposable element families from genomic sequences. Nucleic Acids Res. 37, e78 (2009).

87. Wenke, T. et al. Targeted identification of short interspersed nuclear element families shows their widespread existence and extreme heterogeneity in plant genomes. Plant Cell 23, 3117-3128 (2011)

88. Xu, Z. \& Wang, H. LTR_FINDER: an efficient tool for the prediction of fulllength LTR retrotransposons. Nucleic Acids Res. 35, W265-W268 (2007).

89. Ellinghaus, D., Kurtz, S. \& Willhoeft, U. LTRharvest, an efficient and flexible software for de novo detection of LTR retrotransposons. BMC Bioinform. 9, 18 (2008).

90. Ou, S. \& Jiang, N. LTR_retriever: a highly accurate and sensitive program for identification of long terminal repeat retrotransposons. Plant Physiol. 176, 1410-1422 (2018)

91. Baucom, R. S. et al. Exceptional diversity, non-random distribution, and rapid evolution of retroelements in the B73 maize genome. PLoS Genet. 5, e1000732 (2009).

92. Su, W., Gu, X. \& Peterson, T. TIR-learner, a new ensemble method for TIR transposable element annotation, provides evidence for abundant new transposable elements in the maize genome. Mol. Plant 12, 447-460 (2019).

93. Xiong, W., He, L., Lai, J., Dooner, H. K. \& Du, C. HelitronScanner uncovers a large overlooked cache of Helitron transposons in many plant genomes. Proc. Natl Acad. Sci. USA 111, 10263-10268 (2014). 
94. Kurtz, S. et al. Versatile and open software for comparing large genomes. Genome Biol. 5, R12 (2004).

95. Nattestad, M. \& Schatz, M. C. Assemblytics: a web analytics tool for the detection of variants from an assembly. Bioinformatics 32, 3021-3023 (2016).

96. Li, H. \& Durbin, R. Fast and accurate short read alignment with BurrowsWheeler transform. Bioinformatics 25, 1754-1760 (2009).

97. Wu, T. D., Reeder, J., Lawrence, M., Becker, G. \& Brauer, M. J. GMAP and GSNAP for genomic sequence alignment: enhancements to speed, accuracy, and functionality. Methods Mol. Biol. 1418, 283-334 (2016).

98. Dobin, A. et al. STAR: ultrafast universal RNA-seq aligner. Bioinformatics 29, 15-21 (2013).

99. Romay, M. C. et al. Comprehensive genotyping of the USA national maize inbred seed bank. Genome Biol. 14, R55 (2013).

100. Baseggio, M. et al. Genome-wide association and genomic prediction models of tocochromanols in fresh sweet corn kernels. Plant Genome 12 (2019).

101. Glaubitz, J. C. et al. TASSEL-GBS: a high capacity genotyping by sequencing analysis pipeline. PLoS ONE 9, e90346 (2014).

102. Danecek, P. et al. The variant call format and VCFtools. Bioinformatics 27, 2156-2158 (2011).

103. Kozlov, A. M., Darriba, D., Flouri, T., Morel, B. \& Stamatakis, A. RAxML-NG: a fast, scalable and user-friendly tool for maximum likelihood phylogenetic inference. Bioinformatics 35, 4453-4455 (2019).

104. Wickham, H. ggplot2: Elegant Graphics for Data Analysis. (Springer-Verlag, New York, 2016).

105. Yu, G. S., Zhu, D. K., Guan, H. \& Lam, Y. T.T.Y. ggtree: an R package for visualization and annotation of phylogenetic trees with their covariates and other associated data. Methods Ecol. Evolut. 8, 28-36 (2017).

106. Jombart, T. adegenet: a $\mathrm{R}$ package for the multivariate analysis of genetic markers. Bioinformatics 24, 1403-1405 (2008).

107. Weir, B. S. \& Cockerham, C. C. Estimating F statistics for the analysis of population structure. Evolution 38, 1358-1370 (1984).

108. Pritchard, J. K., Stephens, M. \& Donnelly, P. Inference of population structure using multilocus genotype data. Genetics 155, 945-959 (2000).

109. Earl, D. A. \& VonHoldt, B. M. STRUCTURE HARVESTER: a website and program for visualizing STRUCTURE output and implementing the Evanno method. Conserv. Genet. Resour. 4, 359-361 (2012).

110. Evanno, G., Regnaut, S. \& Goudet, J. Detecting the number of clusters of individuals using the software STRUCTURE: a simulation study. Mol. Ecol. 14, 2611-2620 (2005).

111. Schliep, K. P. phangorn: phylogenetic analysis in R. Bioinformatics 27, 592-593 (2011).

112. Pfeifer, B., Wittelsbürger, U., Ramos-Onsins, S. E. \& Lercher, M. J. PopGenome: an efficient Swiss army knife for population genomic analyses in R. Mol. Biol. Evol. 31, 1929-1936 (2014).

113. Yin, T., Cook, D. \& Lawrence, M. ggbio: an R package for extending the grammar of graphics for genomic data. Genome Biol. 13, R77 (2012).

114. Chen, H., Patterson, N. \& Reich, D. Population differentiation as a test for selective sweeps. Genome Res. 20, 393-402 (2010).

115. Yang, N. et al. Contributions of Zea mays subspecies mexicana haplotypes to modern maize. Nat. Commun. 8, 1874 (2017).

\section{Acknowledgements}

This work was supported by the National Institute of Food and Agriculture (SCRI 201851181-28419 to M.F.R.R., A.M.S., and W.F.T.). We thank Victor Llaca for assistance with the Bionano mapping construction and hybrid assembly.

\section{Author contributions}

Y.H., V.C., and M.F.R.R. designed the research and were responsible for drafting this paper. B.S.F.M., K.L., B.W., L.H., L.C.H., K.S., M.A.G., A.M.M., A.M.S., W.F.T., and M.F.R.R. critically revised the first and subsequent drafts of the paper. Y.H. and M.F.R.R. performed the genome assembly, protein-coding gene annotations, quality assessments, assembly validation, and comparative genomic analysis. Y.H., V.C., and M.F.R.R. performed the population genomic and phylogenomic analyses. K.L., C.F., J.M.G., and E.F.R. isolated the genomic DNA and RNA and performed the flow cytometry experiment. Y.H. and B.S.F.M performed the transposon annotations, B.W. helped on protein-coding gene annotations, and A.N. analyzed Pacbio Isoseq data. C.J.N., E.M.J., T.A.H., A.M.M., and M.F.R.R. analyzed the hyplotypes of sul locus. All of the authors read and approved the paper.

\section{Competing interests}

The authors declare no competing interests.

\section{Additional information}

Supplementary information The online version contains supplementary material available at https://doi.org/10.1038/s41467-021-21380-4.

Correspondence and requests for materials should be addressed to M.F.R.R.Jr.

Peer review information Nature Communications thanks Jeffrey Ross-Ibarra, and the other, anonymous, reviewer(s) for their contribution to the peer review of this work.

Reprints and permission information is available at http://www.nature.com/reprints

Publisher's note Springer Nature remains neutral with regard to jurisdictional claims in published maps and institutional affiliations.

(c) (i) Open Access This article is licensed under a Creative Commons Attribution 4.0 International License, which permits use, sharing, adaptation, distribution and reproduction in any medium or format, as long as you give appropriate credit to the original author(s) and the source, provide a link to the Creative Commons license, and indicate if changes were made. The images or other third party material in this article are included in the article's Creative Commons license, unless indicated otherwise in a credit line to the material. If material is not included in the article's Creative Commons license and your intended use is not permitted by statutory regulation or exceeds the permitted use, you will need to obtain permission directly from the copyright holder. To view a copy of this license, visit http://creativecommons.org/ licenses/by/4.0/.

(C) The Author(s) 2021 